\title{
COMPLEXITY OF PROPOSITIONAL PROOFS UNDER A PROMISE
}

\author{
NACHUM DERSHOWITZ AND IDDO TZAMERET
}

\begin{abstract}
We study - within the framework of propositional proof complexity - the problem of certifying unsatisfiability of CNF formulas under the promise that any satisfiable formula has many satisfying assignments, where "many" stands for an explicitly specified function $\Lambda$ in the number of variables $n$. To this end, we develop propositional proof systems under different measures of promises (that is, different $\Lambda$ ) as extensions of resolution. This is done by augmenting resolution with axioms that, roughly, can eliminate sets of truth assignments defined by Boolean circuits. We then investigate the complexity of such systems, obtaining an exponential separation in the average-case between resolution under different size promises:

(1) Resolution has polynomial-size refutations for all unsatisfiable $3 \mathrm{CNF}$ formulas when the promise is $\varepsilon \cdot 2^{n}$, for any constant $0<\varepsilon<1$.

(2) There are no sub-exponential size resolution refutations for random $3 \mathrm{CNF}$ formulas, when the promise is $2^{\delta n}$ (and the number of clauses is $\left.o\left(n^{3 / 2}\right)\right)$, for any constant $0<\delta<1$.
\end{abstract}

"Goods Satisfactory or Money Refunded"

- The Eaton Promise

\section{INTRODUCTION}

Demonstrating unsatisfiability of propositional formulas is a fundamental problem in both logic and complexity theory, as well as in hardware and software validation. Any standard sound and complete propositional proof system has the ability to separate the set of unsatisfiable formulas in conjunctive normal form (CNF) from the set of CNF formulas having at least one satisfying assignment, in the sense that every unsatisfiable CNF has a refutation in the system, while no satisfiable CNF has one. Our goal is to develop and study, within the framework of propositional proof complexity, systems that are "sound and complete" in a relaxed sense: they can separate

2000 Mathematics Subject Classification. 03F20, 68Q17, 68Q15.

Key words and phrases. Propositional proof complexity, Resolution, Random 3CNF, Promise problems.

This work was carried out in partial fulfillment of the requirements for the Ph.D. degree of the second author and was supported in part by the Israel Science Foundation (grant no. 250/05). 
the set of unsatisfiable CNF formulas from the set of CNF formulas having sufficiently many satisfying assignments (where the term "sufficiently many" stands for an explicitly given function of the number of variables in the $\mathrm{CNF}$ ). We call such proof systems promise refutation systems, as they are complete and sound for the set of CNF formulas promised to be either unsatisfiable or to have many satisfying assignments.

As the proof systems we develop here are intended for proving unsatisfiability of CNF formulas (in other words, to refute them, which is the same as validating their negation), throughout this paper we work solely with refutation systems, and speak about "refutations" and "proofs" interchangeably, always intending refutations, unless otherwise stated. In particular, we work with refutation systems that extend the widely studied resolution refutation system.

Our first task is to introduce a natural model for promise propositional refutation systems. This is accomplished by augmenting standard resolution (or any other propositional proof system extending resolution) with an additional collection of axioms, the promise axioms. Each refutation in a promise refutation system can make use of at most one promise axiom. The promise axioms are meant to capture the idea that we can ignore or "discard" a certain number of truth assignments from the space of all truth assignments, and still be able to certify (due to the promise) whether or not the given CNF is unsatisfiable. The number of assignments that a promise axiom is allowed to discard depends on the promise we are given, and, specifically, it needs to be less than the number of assignments promised to satisfy a given CNF (unless it is unsatisfiable).

Assuming we have a promise that a satisfiable CNF has more than $\Lambda$ satisfying assignments, we can discard up to $\Lambda$ assignments. We refer to $\Lambda$ as the promise. This way, the refutation system is guaranteed not to contain refutations of $\mathrm{CNF}$ formulas having more than $\Lambda$ satisfying assignments, as even after discarding (at most $\Lambda$ ) assignments, we still have at least one satisfying assignment left. On the other hand, any unsatisfiable CNF formula has a refutation in the system, as resolution already has a refutation of it.

We now explain (somewhat informally) what it means to "discard" assignments and how promise axioms formulate the notion of discarding the correct number of truth assignments. Essentially, we say that a truth assignment $\bar{a}$ is discarded by some Boolean formula if $\bar{a}$ falsifies the formula. More formally, let $X:=\left\{x_{1}, \ldots, x_{n}\right\}$ be the set of underlying variables of a given $\mathrm{CNF}$, called the original variables. Let $A$ be some $\mathrm{CNF}$ formula in the variables $X$, and assume that $A$ also contains variables not from $X$, called extension variables. Let $\bar{a} \in\{0,1\}^{n}$ be a truth assignment for the $X$ variables, and assume that there is no extension of $\bar{a}$ (assigning values to the extension variables) that satisfies $A$. Thus, any assignment satisfying $A$ must also satisfy $X \not \equiv \bar{a}$ (that is, $A \models X \not \equiv \bar{a}$ ), and so any (implicationally) complete proof system can prove $X \not \equiv \bar{a}$ from $A$, or, in the case of a 
refutation system, can refute $X \equiv \bar{a}$, given $A$. In this case, we say that the assignment $\bar{a}$ is discarded by $A$.

The promise axioms we present enjoy two main properties:

(1) They discard assignments from the space of possible assignments to the variables $X$.

(2) They express the fact that not too many assignments to the variables $X$ are being discarded (in a manner made precise).

The first property is achieved as follows: Let $C$ be any Boolean circuit with $n$ output bits. Then we can formulate a CNF formula $A$ (using extension variables) expressing the statement that the output of $C$ is (equal to) the vector of variables $X$. This enables $A$ to discard every truth assignment to the variables of $X$ that is outside the image of the Boolean map defined by $C$, because, if an assignment $\bar{a}$ to $X$ is not in the image of $C$, then no extension of $\bar{a}$ can satisfy $A$-assuming the formulation of $A$ is correct. (For technical reasons, the actual definition is a bit different than what is described here; see Section 3.)

The second property is achieved as follows: Assume we can make explicit the statement that the domain of the map defined by the Boolean circuit $C$ above is of size at least $2^{n}-\Lambda$. (See Section 3 for more details.) Then, for the second property to hold, it is sufficient that the axiom formulates the statement that the circuit $C$ defines an injective map (and thus the image of the map contains enough truth assignments), which can be done quite naturally.

Given a certain promise and its associated promise axiom, we call a refutation of resolution, augmented with the promise axiom, a resolution refutation under the (given) promise.

Our second task, besides introducing the model of promise refutation systems, is to investigate the basic properties of this model and in particular to determine its average-case proof complexity with respect to different size of promises (see below for a summary of our findings in this respect).

1.1. Background and Motivation. In propositional proof complexity theory, it is standard to consider an abstract or formal propositional proof system (usually called a Cook-Reckhow proof system, following [CR79]) as a polynomial-time algorithm $A$ that receives a Boolean formula $F$ (usually in CNF) and a string $\pi$ over some finite alphabet ("the (proposed) refutation" of $F$ ), such that there exists a $\pi$ with $A(F, \pi)=1$ if and only if $F$ is unsatisfiable. (A string $\pi$ for which $A(F, \pi)=1$ is also called a witness for the unsatisfiability of $F$.) Equipped with this abstract definition of propositional proof systems, showing that for every abstract proof system there exists some family of formulas $F$ for which there is no polynomially-bounded family of proofs $\pi$ of $F$ is equivalent to showing $\mathbf{N P} \neq \mathbf{c o - N P}$.

For this reason (among others), it is customary in proof complexity theory to concentrate on specific (sometimes provably weaker) proof systems for which proofs have a simple structure. This makes the complexity analysis 
of such proof systems simpler. Prominent examples of such systems are Frege systems and weaker subsystems of Frege, the most notable being the resolution refutation system [Rob65], which also plays an important rôle in many automated theorem provers. In accordance with this, we shall be interested in the present paper not with abstract proof systems (that is, not with finding general witnesses for unsatisfiability, possibly under a promise), but rather with specific and more structured proof systems, and specifically with refutation systems built up as extensions of resolution.

A natural relaxation of the problem of unsatisfiability certification is to require that, if a CNF is satisfiable, then it actually has many satisfying assignments. As mentioned above, we call the specific number of assignments (as a function of the number of variables $n$ ) required to satisfy a satisfiable CNF formula, the "promise". Accordingly, one can define an abstract promise proof system in an analogous manner to the definition of an abstract proof system. It is thus natural to ask whether giving such a promise can help in obtaining shorter proofs of unsatisfiability.

In the case of a big promise, that is, a constant fraction of the space of all truth assignments $\left(\Lambda=\varepsilon \cdot 2^{n}\right.$, for a constant $\left.0<\varepsilon<1\right)$, there is already a deterministic polynomial-time algorithm for any fixed natural number $k$ that certifies the unsatisfiability of all unsatisfiable $k \mathrm{CNF}$ formulas under the promise: The algorithm receives a $k \mathrm{CNF}$ that is either unsatisfiable or has more than $\Lambda$ satisfying assignments and answers whether the formula is unsatisfiable (in case the formula is satisfiable the algorithm provides a satisfying assignment). See [Hir98, Tre04] for such efficient algorithms. ${ }^{1}$ This trivially implies the existence of polynomial-size witnesses for any unsatisfiable $k \mathrm{CNF}$ under the promise $\varepsilon \cdot 2^{n}$. But does resolution already admit such short witnesses of unsatisfiability (that is, resolution refutations) under a big promise? We show that the answer is positive (for all unsatisfiable $3 \mathrm{CNF}$ formulas).

In the case of a smaller promise, by which we mean $\Lambda=2^{\delta n}$ for a constant $0<\delta<1$, it is possible to efficiently transform any CNF over $n$ variables to a new CNF with $n^{\prime}=\lceil n /(1-\delta)\rceil$ variables, such that the original CNF is satisfiable if and only if the new CNF has at least $2^{\delta n^{\prime}}$ satisfying assignments. ${ }^{2}$ Thus, the worst-case complexity of certifying CNF unsatisfiability under such a promise is polynomially equivalent to the worst-case complexity of certifying CNF unsatisfiability without a promise. However, it is still possible that a promise of $2^{\delta n}$ might give some advantage (that is, a superpolynomial speedup over refutations without a promise) in certifying the

\footnotetext{
${ }^{1}$ In the case the promise is $\Lambda=2^{n} / \operatorname{poly}(n)$, the algorithm in [Hir98] also gives a deterministic sub-exponential time procedure for unsatisfiability certification of $k \mathrm{CNF}$ formulas (for a constant $k$ ).

2 This can be achieved simply by adding new $\left(n^{\prime}-n\right)$ "dummy variables". For instance, by adding the clauses of a tautological CNF in these dummy variables to the original CNF. This way, if the original CNF has at least one satisfying assignment then the new CNF has at least $2^{n^{\prime}-n} \geq 2^{\delta n^{\prime}}$ satisfying assignments.
} 
unsatisfiability of certain (but not all) CNF formulas; for instance, in the average-case. $^{3}$

Feige, Kim, and Ofek [FKO06] have shown that when the number of clauses is $\Omega\left(n^{7 / 5}\right)$ there exist polynomial-size witnesses to the unsatisfiability of $3 \mathrm{CNF}$ formulas in the average-case. On the other hand, Beame, Karp, Pitassi, and Saks [BKPS02] and Ben-Sasson and Wigderson [BSW01] showed that resolution does not provide sub-exponential refutations for $3 \mathrm{CNF}$ formulas in the average-case when the number of clauses is at most $n^{(3 / 2)-\epsilon}$, for any constant $0<\epsilon<1 / 2 .{ }^{4}$ This shows that general witnessing of $3 \mathrm{CNF}$ unsatisfiability is strictly stronger than resolution refutations. But is it possible that, under a promise of $2^{\delta n}$, resolution can do better in the average-case? We show that the answer is negative.

There are two main motivations for studying propositional proofs under a given promise and their complexity. The first is to answer the natural question whether CNF unsatisfiability certification enjoys any advantage given a certain promise. As already mentioned, the answer is positive when the promise is a constant fraction of all the truth assignments, and our results imply that this phenomenon already occurs for resolution. For a small promise of $2^{\delta n}$, we can show that, at least in the case of resolution refutations of most $3 \mathrm{CNF}$ formulas (of certain clause-to-variable density), the answer is negative. In fact, we can show that the answer stays negative even when the promise is bigger than $2^{\delta n}$, and specifically when $\Lambda=2^{n} / 2^{n^{\xi}}$ for some constant $0<\xi<1$. Overall, our results establish the first unsatisfiability certification model in which a promise of a certain given size is known to help (that is, allow more efficient certifications) in the average-case, while promises of smaller sizes do not help.

The second motivation is more intrinsic to proof complexity theory: It is a general goal to develop natural frameworks for propositional proofs that are not sound in the strict sense, but rather possess an approximate notion of soundness (like showing that certain "approximations" give speed-ups). For this purpose, the proof systems we propose formalize - in a natural waythe notion of separating unsatisfiable CNF formulas from those that have many satisfying assignments. The promise axioms we present also allow for a natural way of controlling the size of the promise, which in addition leads to an exponential separation between different size promises.

\footnotetext{
${ }^{3}$ Note that if we add dummy variables to a $3 \mathrm{CNF}$ then we obtain an "atypical instance" of a $3 \mathrm{CNF}$. Thus, assuming we have polynomial-size witnesses of unsatisfiability of $3 \mathrm{CNF}$ formulas under a small promise in the average-case (that is, the "typical case"), the reduction alone (that is, adding dummy variables) does not automatically yield polynomial-size witnesses for 3CNF formulas in the average-case without a promise as well.

${ }^{4}$ Beame et al. [BKPS02] showed such a lower bound for $n^{(5 / 4)-\epsilon}$ number of clauses (for any constant $0<\epsilon<1 / 4$ ). Ben-Sasson and Wigderson [BSW01] introduced the size-width tradeoff that enables one to prove an exponential lower bound for random 3CNF formulas with $n^{(3 / 2)-\epsilon}$ number of clauses (for any constant $0<\epsilon<1 / 2$ ), but the actual proof for this specific clause-number appears in [BS01].
} 
This paper introduces the concept of propositional proofs under a promise, analyzes the proof complexity of these proof systems with respect to different promise sizes, giving a separation between promises of different sizes, and also illustrates several new facts about the widely studied resolution proof system.

1.2. Results. We show that resolution refutations are already enough to efficiently separate unsatisfiable 3CNF formulas from those 3CNF formulas with an arbitrarily small constant fraction of satisfying assignments. In particular, in Section 4, we show the following:

Main Result 1: Let $0<\varepsilon<1$ be any constant and let $\Lambda=\varepsilon \cdot 2^{n}$ be the given promise. Then every unsatisfiable $3 \mathrm{CNF}$ with $n$ variables has a polynomialsize (in $n$ ) resolution refutation under the promise $\Lambda$.

The proof of this resembles a deterministic algorithm of Trevisan [Tre04] for approximating the number of satisfying assignments of $k \mathrm{CNF}$ formulas.

In contrast to the case of a big promise, the results show that, at least for resolution, a small promise of $\Lambda=2^{\delta n}$ (for any constant $0<\delta<1$ ) does not give any advantage over standard resolution (that is, resolution without the promise axioms) in most cases (that is, in the average-case). Specifically, in Section 5 we show the following:

Main Result 2: Let $0<\delta<1$ be any constant and let $\Lambda=2^{\delta n}$ be the given promise. Then, there is an exponential lower bound on the size of resolution refutations of random $3 \mathrm{CNF}$ formulas under the promise $\Lambda$, when the number of clauses is $o\left(n^{3 / 2}\right)$.

This lower bound actually applies to a more general model of promise proofs. It remains valid even if we allow (somehow) the promise proofs to discard arbitrarily chosen sets of truth assignments (of size $\Lambda=2^{\delta n}$ ), and not necessarily those sets that are definable by (small) Boolean circuits. In fact, the lower bound applies even to a bigger promise of $\Lambda=2^{n-n^{\xi}}$, for some constant $0<\xi<1$.

The proof strategy for this lower bound follows that of Ben-Sasson and Wigderson [BSW01] (the size-width tradeoff approach), and so the rate of the lower bound matches the one in that paper. The main novel observation is that under the appropriate modifications this strategy also works when one restricts the set of all truth assignments to a smaller set (that is, from $2^{n}$ down to $2^{n}-2^{\delta n}$ for a constant $0<\delta<1$, and in fact down to $2^{n}-2^{n} / 2^{n^{\xi}}$, for some constant $0<\xi<1$ ).

It is important to note that these two main results show that the decision to discard sets of truth assignments defined by Boolean circuits does not affect the results in any way, and thus should not be regarded as a restriction of the model of promise refutations (at least not for resolution). To see this, note that we could allow a promise refutation to discard arbitrarily chosen sets of truth assignments (of the appropriate size determined by the given promise), that is, sets of truth assignments that are not necessarily 
definable by (small) Boolean circuits. However, although this modification strengthens the model, it is not really necessary for the upper bound in Main Result 1, as this upper bound is already valid when one discards sets of truth assignments by (small) Boolean circuits. On the other hand, as mentioned above, the lower bound in Main Result 2 is already valid when one allows a promise refutation to discard any arbitrarily chosen set of truth assignments (of the appropriate size).

The exact model of promise propositional proof systems is developed in Section 3. It is preceded, in the next section, by preliminaries and terminological conventions.

\section{Preliminaries}

2.1. Notations. For natural number $m$, we use $[m]$ to denote the set $\{1, \ldots, m\}$ of naturals.

Let $A, B$ be two propositional formulas. We write $A \equiv B$ as an abbreviation for $(A \rightarrow B) \wedge(B \rightarrow A)$. The notation $A \not \equiv B$ abbreviates $\neg(A \equiv B)$. We say that $A$ semantically implies $B$, denoted by $A \models B$, iff every satisfying assignment to $A$ also satisfies $B$.

A CNF formula over the variables $x_{1}, \ldots, x_{n}$ is defined as follows: A literal is a variable $x_{i}$ or its negation $\neg x_{i}$. A clause is a disjunction of literals. We treat a clause as a set of literals, that is, we delete multiple occurrences of the same literal in a clause. A CNF formula is a conjunction of clauses (sometimes treated also as a set of clauses, where the conjunction between these clauses is implicit). A $k \mathrm{CNF}$ formula is a CNF with all clauses containing $k$ literals each.

The width of a clause $D$ is the number of literals in it, denoted $|D|$. The size of a CNF formula $K$ is the total number of clauses in it, denoted $|K|$. The width of a CNF formula $K$ is the maximum width of a clause in $K$.

We denote by $K^{\prime} \subseteq K$ that $K^{\prime}$ is a sub-collection of clauses from $K$.

2.2. Resolution Refutation Systems. Resolution is a complete and sound proof system for unsatisfiable CNF formulas.

Let $C$ and $D$ be two clauses containing neither $x_{i}$ nor $\neg x_{i}$. The resolution rule allows one to derive $C \vee D$ from $C \vee x_{i}$ and $D \vee \neg x_{i}$. The clause $C \vee D$ is called the resolvent of the clauses $C \vee x_{i}$ and $D \vee \neg x_{i}$ on the variable $x_{i}$, and we also say that $C \vee x_{i}$ and $D \vee \neg x_{i}$ were resolved over $x_{i}$.

The weakening rule allows one to derive the clause $C \vee D$ from the clause $C$, for any two clauses $C, D$.

Definition 2.1 (Resolution). A resolution proof of the clause $D$ from a $C N F$ formula $K$ is a sequence of clauses $D_{1}, D_{2}, \ldots, D_{\ell}$, such that: (1) each clause $D_{j}$ is either a clause of $K$ or a resolvent of two previous clauses in the sequence or derived by the weakening rule from a previous clause in the sequence; (2) the last clause $D_{\ell}=D$. The size of a resolution proof is the total number of clauses in it. The width of a resolution proof is the maximal width of a clause in it. A resolution refutation of a CNF formula $K$ is a 
resolution proof of the empty clause $\square$ from $K$. (The empty clause stands for FALSE; that is, the empty clause has no satisfying assignments.)

Let $K$ be an unsatisfiable CNF formula. The resolution refutation size of $K$ is the minimal size of a resolution refutation of $K$ and is denoted $S(K)$. Similarly, the resolution refutation width of $K$ is the minimal width of a resolution refutation of $K$ and is denoted $w(K)$. If $K$ has a polynomial-size resolution refutation we say that resolution can efficiently certify the unsatisfiability of $K$. Similarly, if the clause $D$ has a polynomial-size resolution proof from $K$ we say that $D$ is efficiently provable from $K$.

2.3. Size-Width Tradeoffs. We recall now the approach for proving size lower bounds on resolution refutations developed by Ben-Sasson and Wigderson [BSW01]. The basic idea is that a lower bound on the resolution refutation width of a CNF formula $K$ implies a lower bound on the resolution refutation size of $K$ :

Theorem 1 ([BSW01]). Let $K$ be a CNF formula of width $r$, then

$$
S(K)=\exp \left(\Omega\left(\frac{(w(K)-r)^{2}}{n}\right)\right) .
$$

2.4. Boolean Circuit Encoding. The promise axioms we introduce use Boolean circuits to define the set of assignments to be discarded (see Section 3). Therefore, as resolution operates only with clauses, we need to encode Boolean circuits as collections of clauses (CNF formulas). We assume that all Boolean circuits use only three gates: $\vee, \wedge, \neg$ (though this is not necessary) where $\vee$ (denoting OR) and $\wedge$ (denoting AND) have fan-in 2 and $\neg$ (denoting NOT) has fan-in 1 . Let $C$ be a Boolean circuit with $m$ input bits and $n$ output bits. Let $\bar{W}=\left\{w_{1}, \ldots, w_{m}\right\}$ be the $m$ input variables of $C$ and let $X$ denote the $n$ variables $\left\{x_{1}, \ldots, x_{n}\right\}$. We consider the $n$ output bits of $C$ as the outputs of $n$ distinct circuits $C_{1}(\bar{W}), \ldots, C_{n}(\bar{W})$ in the $\bar{W}$ variables, and we write $C(\bar{W}) \equiv X$ to mean that $X$ equals the output of $C(\bar{W})$ (that is, $\left.C_{1}(\bar{W}) \equiv x_{1} \wedge \cdots \wedge C_{n}(\bar{W}) \equiv x_{n}\right)$. This notation can be extended in a similar manner to $C\left(\overline{W_{1}}\right) \equiv C^{\prime}\left(\overline{W_{2}}\right)$ and $C\left(\overline{W_{1}}\right) \not \equiv C^{\prime}\left(\overline{W_{2}}\right)$.

By Cook's Theorem, there exists a CNF formula $F$ (in both the $\bar{W}$ variables and new extension variables) that encodes the circuit $C$. This means that there are $n$ new extension variables (among other extension variables) $y_{1}, \ldots, y_{n}$ in $F$ such that for all assignments $\bar{a}: \quad F(\bar{a})=1$ iff $C\left(w_{1}(\bar{a}), \ldots, w_{m}(\bar{a})\right)=y_{1}(\bar{a}) \circ \cdots \circ y_{n}(\bar{a})$, where we denote by $w_{i}(\bar{a})$ the truth value of $w_{i}$ under the assignment $\bar{a}$ and by $\circ$ the concatenation of Boolean bits. In other words, $F$ expresses the fact that $y_{1}, \ldots, y_{n}$ are the output bits of $C$. If $C$ is of size $s$ (that is, the number of Boolean gates in $C$ is $s$ ), then the size of $F$ is $O(s \cdot \log (s))$. Therefore, if $C$ is of size polynomial in $n$ then $F$ is also of polynomial-size in $n$. We denote by $\|C(\bar{W})\|$ the CNF formula $F$ that encodes $C(\bar{W})$.

For most purposes, we will not need an explicit description of how the encoding of Boolean circuits as CNF formulas is done through $\|C(\bar{W})\|$. 
Nevertheless, in Section 4 we need to ensure that resolution can efficiently prove several basic facts about the encoded circuits. For this reason, and for the sake of concreteness of the promise axioms (Definitions 3.3 and 3.4) we provide the precise definition of the encoding in the Appendix (Section A.1), in addition to proving some of the encoding's basic (proof theoretical) properties. The interested reader can look at the Appendix for any missing details, but anyone willing to accept the existence of an efficient CNF encoding of Boolean circuits that is also intensional for resolution (in the sense that resolution can efficiently prove basic properties of the encoded circuits) can skip Section A.1 without risk.

\section{Promise Proof Systems}

In this section we define precisely the model of refutations under a promise. As discussed in the introduction, we work with the resolution refutation system as our underlying system and augment it with a new set of axioms that we call the promise axioms. We call this proof system promise resolution. The promise axioms are meant to express the fact that we can discard a certain number of truth assignments from the space of all truth assignments and still be able to certify (due to the promise) whether the input $\mathrm{CNF}$ is unsatisfiable or not. Each promise resolution refutation can use at most one promise axiom.

From now on, throughout the paper, we shall assume that the underlying variables of the CNF formulas that are meant to be refuted are taken from the set $X:=\left\{x_{1}, \ldots, x_{n}\right\}$. The $X$ variables are called the original variables. Any other variable that appears in a (promise resolution) refutation is called an extension variable.

Definition 3.1 (CNF formulas under a promise). Let $\Lambda$ be a fixed function in $n$ (the number of $X$ variables) such that $0 \leq \Lambda(n) \leq 2^{n}$. The function $\Lambda$ is called the promise. The set of $C N F$ formulas under the promise $\Lambda$ consists of all CNF formulas in the $X$ variables that are either unsatisfiable or have more then $\Lambda(n)$ satisfying assignments (for $n=|X|$ ).

The refutation systems we build are sound and complete for the set of CNF formulas under a (given) promise. That is, every unsatisfiable CNF formula has a refutation in the system (this corresponds to completeness), while no CNF having $n$ variables and more than $\Lambda(n)$ satisfying assignments has a refutation in it (this corresponds to soundness under the promise). Soundness (under the promise) is achieved by requiring that resolution should prove the fact that we discard the right number of assignments (see Section 3.1 for details).

Definition 3.2 (Assignment discarding). Let $A$ be a CNF in the $X$ variables that can contain (but does not necessarily contain) extension variables (that is, variables not from $X$ ). We say that an assignment to the $X$ variables $\bar{a}$ is discarded by $A$ if there is no extension of $\bar{a}$ (to the extension variables in $A)$ that satisfies $A$. 
(See Section 1 for more regarding assignment discarding.)

\subsection{Promise Axioms.}

3.1.1. Big promise. We first concentrate on a promise of a constant fraction of assignments (for a smaller promise the axiom is similar; see below).

Let the promise (see Definition 3.1) be $\Lambda=\varepsilon \cdot 2^{n}$, for a constant $0<$ $\varepsilon<1$ (we fix this $\Lambda$ throughout this subsection), and let $r=\lceil\log (1 / \varepsilon)\rceil$ and $t=2^{r}-1$. Let $C$ be a sequence of Boolean circuits $C:=\left(C^{(1)}, \ldots, C^{(t)}\right)$. Assume that each $C^{(i)}$ has $n-r$ input bits and $n$ output bits and computes the Boolean map $f_{i}:\{0,1\}^{n-r} \rightarrow\{0,1\}^{n}$. Assume further that the $f_{i}$ 's are all injective maps and that the images of all these maps are pairwise disjoint. Denote by $\operatorname{Im}\left(f_{i}\right)$ the image of the map $f_{i}$. For simplicity, we call the union $\cup_{i=1}^{t} \operatorname{Im}\left(f_{i}\right)$ the image of $C$ and denote it by $\operatorname{Im}(C)$. By the definition of $r$, we have $2^{n-r} \leq \varepsilon \cdot 2^{n}$, and by the injectivity and pairwise disjointness of the images of the $f_{i}$ 's we have:

$$
|\operatorname{Im}(C)|=t \cdot 2^{n-r}=\left(2^{r}-1\right) \cdot 2^{n-r}=2^{n}-2^{n-r} \geq 2^{n}-\Lambda .
$$

Therefore, we can treat $\operatorname{Im}(C)$ as the set of all possible truth assignments for the original variables $X$, without losing soundness: If $K$ is unsatisfiable then there is no assignment in $\operatorname{Im}(C)$ that satisfies $K$; and if $K$ is satisfiable then according to the promise it has more than $\Lambda$ satisfying assignments, which means that there is at least one assignment in $\operatorname{Im}(C)$ that satisfies $K$. This idea is formulated as a propositional formula as follows:

Definition 3.3 (Promise Axiom for $\Lambda=\varepsilon \cdot 2^{n}$ ). Let the promise be $\Lambda=\varepsilon \cdot 2^{n}$, for a constant $0<\varepsilon<1$, and let $r=\lceil\log (1 / \varepsilon)\rceil$ and $t=2^{r}-1$. Let $C$ be a sequence of Boolean circuits $C:=\left(C^{(1)}, \ldots, C^{(t)}\right)$. Assume that each $C^{(i)}$ has $n-r$ input bits and $n$ output bits and let $\overline{W_{1}}$ and $\overline{W_{2}}$ be two disjoint sets of $n-r$ extension variables each. The promise axiom $P R M_{C, \Lambda}$ is the CNF encoding (via the encoding defined in Section A.1) of the following Boolean formula:

$$
\begin{array}{r}
\left(\bigwedge_{i=1}^{t}\left(C^{(i)}\left(\overline{W_{1}}\right) \equiv C^{(i)}\left(\overline{W_{2}}\right) \rightarrow \overline{W_{1}} \equiv \overline{W_{2}}\right) \wedge \bigwedge_{1 \leq i<j \leq t} C^{(i)}\left(\overline{W_{1}}\right) \not \equiv C^{(j)}\left(\overline{W_{2}}\right)\right) \\
\longrightarrow \bigvee_{i=1}^{t} C^{(i)}\left(\overline{W_{1}}\right) \equiv X .
\end{array}
$$

The promise axiom $\mathrm{PRM}_{C, \Lambda}$ expresses the fact that if each circuit in $C$ computes an injective map (this is formulated as $\wedge_{i=1}^{t}\left(C^{(i)}\left(\overline{W_{1}}\right) \equiv\right.$ $\left.C^{(i)}\left(\overline{W_{2}}\right) \rightarrow \overline{W_{1}} \equiv \overline{W_{2}}\right)$ ), and if the images of the maps computed by each pair of circuits in $C$ are disjoint (this is formulated as $\wedge_{1 \leq i<j \leq t} C^{(i)}\left(\overline{W_{1}}\right) \not \equiv$ $C^{(j)}\left(\overline{W_{2}}\right)$ ), then we can assume that the assignments to the original variables $X$ are taken from the image of $C$ (this is formulated as $\vee_{i=1}^{t} C^{(i)}\left(\overline{W_{1}}\right) \equiv X$ ). The fact that the image of $C$ is of size at least $2^{n}-\Lambda$ is expressed (due to Equation (1)) by the number of input bits (that is, $n-r$ ) of each circuit in $C$ and the number of circuits in $C$ (that is, $t$ ). Also note that the promise 
axiom is of polynomial-size as long as the circuits in $C$ are (since $1 / \varepsilon$ is a constant).

The following claim shows that the promise axioms are sound with respect to the promise $\Lambda$, in the sense that they do not discard too many truth assignments:

Claim 1. The promise axiom $P R M_{C, \Lambda}$ discards at most $\Lambda$ truth assignments. That is, there are at most $\Lambda$ distinct assignments $\bar{a}$ to the $X$ variables such that $P R M_{C, \Lambda} \models X \not \equiv \bar{a}$.

Proof. Assume that some Boolean map computed by some circuit in $C$ is not injective. Then any assignment to the $X$ variables has an extension $\rho$ (to the extension variables in the promise axiom) that falsifies the premise of the main implication in $\mathrm{PRM}_{C, \Lambda}$ and thus $\rho$ satisfies $\mathrm{PRM}_{C, \Lambda}$. Therefore no assignments to $X$ are discarded.

Similarly, assume that the images of some pair of maps computed by two circuits in $C$ are not disjoint. Then, again, any assignment to the $X$ variables has an extension that satisfies $\mathrm{PRM}_{C, \Lambda}$, and so no assignments to $X$ are discarded.

Assume that all the Boolean maps computed by circuits in $C$ are injective and have pairwise disjoint images. Then every assignment satisfies the premise of the main implication in the promise axiom $\mathrm{PRM}_{C, \Lambda}$. Therefore, it suffices to show that the consequence of the main implication of the axiom (that is, $\left.\vee_{i=1}^{t} C^{(i)}\left(\overline{W_{1}}\right) \equiv X\right)$ discards at most $\Lambda$ assignments to the $X$ variables. By definition (of the encoding of the circuits) for all assignments $\bar{a}$ to the $X$ variables that are in $\operatorname{Im}(C)$ there is an extension of $\bar{a}$ that satisfies $\vee_{i=1}^{t} C^{(i)}\left(\overline{W_{1}}\right) \equiv X$. Now, all the circuits $C^{(i)}$ compute injective maps with pairwise disjoint images, and thus by Equation (1) there are at least $2^{n}-\Lambda$ distinct elements (that is, assignments) in $\operatorname{Im}(C)$. Hence, at least $2^{n}-\Lambda$ assignments to the $X$ variables are not discarded.

3.1.2. Smaller promise. We shall also need to formulate promise axioms for promises smaller than $\varepsilon \cdot 2^{n}$. Specifically, we shall work with a promise of $\Lambda=2^{\delta n}$ for a constant $0<\delta<1$ (we fix this $\Lambda$ throughout this subsection). For such a promise, the promise axiom is similar to Definition 3.3, except that the number of input bits of each circuit in $C$ needs to be modified accordingly. (We shall use the same terminology as that used above for the Big Promise.)

Definition 3.4 (Promise Axiom for $\Lambda=2^{\delta n}$ ). Let the promise be $\Lambda=2^{\delta n}$, for a constant $1<\delta<1$, and let $t=\lceil(1-\delta) n\rceil$. Let $C$ be a sequence of Boolean circuits $C:=\left(C^{(1)}, \ldots, C^{(t)}\right)$. Assume that for each $1 \leq i \leq t$ the circuit $C^{(i)}$ has $n-i$ input bits and $n$ output bits. Let $\overline{W_{1}}, \ldots, \overline{W_{t}}$ and $\overline{W_{1}^{\prime}}, \ldots, \overline{W_{t}^{\prime}}$ be $2 t$ disjoint sets of extension variables ${ }^{5}$, where for all $1 \leq i \leq t$,

\footnotetext{
${ }^{5}$ We have not been very economical in adding extension variables here; but this is not essential.
} 
$W_{i}, W_{i}^{\prime}$ consist of $n-i$ variables each. The promise axiom $P R M_{C, \Lambda}$ is the CNF encoding (via the encoding defined in Section A.1) of the following Boolean formula:

$$
\begin{array}{r}
\left(\bigwedge_{i=1}^{t}\left(C^{(i)}\left(\overline{W_{i}}\right) \equiv C^{(i)}\left(\overline{W_{i}^{\prime}}\right) \rightarrow \overline{W_{i}} \equiv \overline{W_{i}^{\prime}}\right) \wedge \bigwedge_{1 \leq i<j \leq t} C^{(i)}\left(\overline{W_{i}}\right) \not \equiv C^{(j)}\left(\overline{W_{j}}\right)\right) \\
\longrightarrow \bigvee_{i=1}^{t} C^{(i)}\left(\overline{W_{i}}\right) \equiv X .
\end{array}
$$

Note that the promise axiom is of polynomial size as long as the circuits in $C$ are (since $t \leq n$ ).

Also note that the proof of Claim 1 did not use the parameters $r$ and $t$ (which determine the number of input bits in the circuits in $C$ and the number of circuits in $C$, respectively) but only the size $|\operatorname{Im}(C)|$. Thus, the same claim holds also for the promise axiom in Definition 3.4, which means that this promise axiom discards at most $2^{n}-|\operatorname{Im}(C)|$ truth assignments, for some sequence of circuits in $C$ that compute injective maps with pairwise disjoint images. Therefore, we need to verify that $|\operatorname{Im}(C)| \geq 2^{n}-\Lambda$, for all $C$ that consists of circuits computing injective maps with pairwise disjoint images.

Notice that for all $1 \leq i \leq t$ the circuit $C^{(i)}$ computes a Boolean map, denoted $f_{i}$, such that $f_{i}:\{0,1\}^{n-i} \rightarrow\{0,1\}^{n}$. Assume that all the $f_{i}$ 's are injective and that the images of each pair of functions $f_{i}, f_{j}$, for $1 \leq i \neq j \leq$ $t$, are disjoint. Then, we have:

$$
\begin{aligned}
|\operatorname{Im}(C)| & =\left(\frac{1}{2}+\frac{1}{2^{2}}+\frac{1}{2^{3}}+\cdots+\frac{1}{2^{t}}\right) \cdot 2^{n}=\left(1-\frac{1}{2^{t}}\right) \cdot 2^{n} \\
& =2^{n}-2^{n-\lceil(1-\delta) n\rceil} \geq 2^{n}-2^{\delta n}=2^{n}-\Lambda
\end{aligned}
$$

Also note that $|\operatorname{Im}(C)| \leq 2^{n}-2^{\delta n-1}$ and so if the circuit in $C$ are injective with pairwise disjoint images then $\mathrm{PRM}_{C, \Lambda}$ discards at least $2^{\delta n} / 2$ truth assignments.

\subsection{Promise Resolution.}

Definition 3.5 (Promise resolution). Let $\Lambda$ be the promise (see Definition 3.1) and let $K$ be a CNF in the $X$ variables. A promise resolution (under the promise $\Lambda$ ) proof of the clause $D$ from a CNF formula $K$ is a sequence of clauses $D_{1}, D_{2}, \ldots, D_{\ell}$ such that:

(1) Each clause $D_{j}$ is either a clause of $K$ or a clause of a promise axiom $P R M_{C, \Lambda}$ (where $P R M_{C, \Lambda}$ is either a big or a smaller promise axiom as defined in Definitions 3.3 and 3.4 and $C$ is an arbitrary sequence of circuits with the prescribed input and output number of bits) or a resolvent of two previous clauses in the sequence;

(2) The sequence contains (the clauses of) at most one promise axiom;

(3) The last clause $D_{\ell}=D$. 
The size, width and refutations of promise resolution is defined the same as in resolution.

Note that promise resolution is a Cook-Reckhow proof system (see the first paragraph in Section 1.1 for a definition): It is possible to efficiently verify whether a given CNF is an instance of the promise axiom, and hence to verify whether a sequence of clauses constitute a legitimate promise refutation. This can be done by "decoding" the CNF that encodes the promise axiom $\mathrm{PRM}_{C, \Lambda}$ and then checking that each circuit in $C$ has the right number of input and output bits (we discuss this issue in some more detail in the appendix).

Proposition 1. Let $\Lambda$ be the promise (where $\Lambda$ is either $\varepsilon \cdot 2^{n}$ or $2^{\delta n}$, for $0<\varepsilon, \delta<1)$. Then promise resolution under the promise $\Lambda$ is a sound and complete proof system for the set of CNF formulas under the promise $\Lambda$ (see Definition 3.1). In other words, every unsatisfiable CNF has a promise resolution refutation and every $C N F$ that has more than $\Lambda$ satisfying assignments does not have promise resolution refutations.

Proof. Completeness stems from completeness of resolution. Soundness under the promise $\Lambda$ stems from Claim 1 (which, by the notes after Definition 3.4 , holds for both the big and the smaller promise axioms).

3.3. Discussion. Let $K$ be an unsatisfiable CNF formula in $n$ variables, $\mathrm{PRM}_{C, \Lambda}$ a promise axiom (where the circuits in $C$ all compute injective and pairwise disjoint Boolean maps) and let $S:=\operatorname{Im}(C) \subseteq\{0,1\}^{n}$ (such that $\left.|S| \geq 2^{n}-\Lambda\right)$. Then, one can think of a promise resolution refutation of $K$ using the axiom $\mathrm{PRM}_{C, \Lambda}$ as containing two separate parts:

(i) a resolution 'refutation' of $K$ where the space of truth assignments is restricted to $S$;

(ii) a resolution proof that $|S| \geq 2^{n}-\Lambda$.

Note that if we want to consider promise resolution as having only part (i), then we can modify (actually, strengthen) the promise axiom into $\vee_{i=1}^{t} C^{(i)}(\bar{W}) \equiv X$. However, this choice means losing the soundness of the proof system under the promise (that is, the soundness with respect to CNF formulas under a promise as defined in Definition 3.1), since we do not have any guarantee that the circuit $C$ discards at most $\Lambda$ assignments (and so CNF formulas with more than $\Lambda$ satisfying assignments might have refutations in such a system).

It is possible to use any number of axioms of the form $C^{(i)}(\bar{W}) \equiv X$, as long as resolution can prove both the injectivity of each of the maps computed by the circuits $C^{(i)}$ introduced and the pairwise disjointness of these maps (as formulated by a propositional formula similar to the formulation in the promise axioms), and provided that the circuits $C^{(i)}$ have number of input bits that induce the right size of domains (that is, that the total size of their domains is at least $2^{n}-\Lambda$ ). 
It is also possible to modify the promise axioms to suit any chosen size of promise $\Lambda$ (possibly, only an approximation of $\Lambda$ ). This can be achieved by choosing a sequence of circuits with the appropriate size of domain (explicitly expressed by the number of input bits in each circuit in the sequence, and the total number of circuits).

Some comments about the formulation of the promise axioms are in order.

Comment 1. Note that we could not use only a single circuit $C$ in the promise axioms (in contrast to a sequence of circuits), because that way we would not have the possibility of controlling the size of the domain of $C$ and efficiently verifying that this size is the correct one inside resolution. To see this, note that if the number of input variables to $C$ is $n$ (the number of original variables) and the map computed by $C$ is (provably) injective then $C$ does not discard any assignment. If, on the other hand, the number of input variables to $C$ is less than $n$, then $C$ discards at least half the truth assignments, which might be too many.

Comment 2. Also note that in order to discard assignments we cannot use a seemingly more natural axiom of the form $C(\bar{W}) \not \equiv X$ for some circuit $C$ (with domain of size $\Lambda$ ). The reason is that this would not discard assignments in the image of $C$ : It is not necessarily true that $C(\bar{W}) \not \equiv X \models X \not \equiv \bar{b}$ for all $\bar{b} \in\{0,1\}^{n}$ such that $b \in \operatorname{Im}(C)$ (notice that even for such a $\bar{b}$ there might be some assignment $\bar{a}$ for which $C(\bar{a}) \not \equiv \bar{b})$.

On the other hand, Jan Krajíček [Kra07] observed that it is possible to discard assignments by an axiom of the form $C(\bar{W}) \not \equiv X$, where $C$ is a fixed circuit with domain of size at most $\Lambda$ (that is, it has $k<n$ number of input bits, where $2^{k} \leq \Lambda$ ), and where the rule of using this axiom is that we can introduce any instance of $C(\bar{W}) \not \equiv X$ where all the variables in $\bar{W}$ are substituted by constants 0,1 and variables from $X$. This choice of axioms simplifies somewhat the actual formulation of the promise axioms, as it does not require that $C$ computes an injective Boolean map. However, a possible drawback of such a formulation is the following: It is possible that for certain circuits (of the appropriate number of input and output bits) we shall need to use exponentially many such axiom instances to discard all (or most of) the assignments pertaining to the image of the circuits. In contrast to this, our formulation of the promise axioms above enables a single instance of a promise axiom using any circuit (more correctly, a sequence of circuits of the appropriate number of input and output bits) to discard all the assignments outside the image of the circuit.

\section{Big Promise: Upper Bound}

In this section, we show that under the promise $\Lambda=\varepsilon \cdot 2^{n}$, for any constant $0<\varepsilon<1$, resolution can efficiently certify the unsatisfiability of all unsatisfiable $3 \mathrm{CNF}$ formulas. The proof method resembles the algorithm

presented by Trevisan [Tre04]. For a constant $k$, this algorithm receives a 
$k \mathrm{CNF}$ formula $K$ and deterministically approximates the fraction of satisfying assignments of $K$ within an additive error of $\varepsilon$. The running time of the algorithm is linear in the size of $K$ and polynomial in $1 / \varepsilon$.

The idea behind the refutations in this section is based on the following observation: Given an unsatisfiable $3 \mathrm{CNF}$ formula $K$ and a constant $c$, either there are $3(c-1)$ variables that hit ${ }^{6}$ all the clauses in $K$ or there are at least $c$ clauses in $K$ over $3 c$ distinct variables denoted by $K^{\prime}$ (that is, each variable in $K^{\prime}$ appears only once). In the first case, we can consider all the possible truth assignments to the $3 c$ variables inside resolution: if $K$ is unsatisfiable then any such truth assignment yields an unsatisfiable 2CNF formula, which can be efficiently refuted in resolution (cf. [Coo71]). In the second case, we can make use of a promise axiom to efficiently refute $K^{\prime}$ (this set of clauses has less then $\Lambda$ satisfying assignments, for sufficiently large $c$ ). Specifically, in the second case, we construct a sequence of small circuits $C$ for which any satisfying assignment for $K^{\prime}$ is provably in resolution (with polynomial-size proofs) outside the image of $C$.

The following is the main result of this section:

Theorem 2. Let $0<\varepsilon<1$ be a constant and let $\Lambda=\varepsilon \cdot 2^{n}$ be the given promise. Then every unsatisfiable $3 C N F$ with $n$ variables has a polynomialsize (in $n$ ) resolution refutation under the promise $\Lambda$.

This theorem is a consequence of the three lemmas that follow.

Lemma 3. Let $K$ be a $3 C N F$ formula. For every integer $c$ one of the following holds: (i) there is a set of at most $3(c-1)$ variables that hit all the clauses in $K$; or (ii) there is a sub-collection of clauses from $K$, denoted $K^{\prime}$, with at least c clauses and where each variable appears only once in $K^{\prime}$.

Proof. Assume that $c>2$ (otherwise the lemma is trivial). Suppose that there is no set of at most $3(c-1)$ variables that hit all the clauses in $K$ and let $D_{1}$ be some clause in $K$. Then, there ought to be a clause $D_{2}$ from $K$ that contains 3 variables that are not already in $D_{1}$ (or otherwise, the 3 (distinct) variables in $D_{1}$ hit all the clauses in $K$, which contradicts the assumption). In a similar manner we can continue to add new clauses from $K$ until we reach a set of $c$ clauses $D_{1}, D_{2}, \ldots, D_{c}$, where no variable appears more than once in this set of clauses.

If case (i) of the prior lemma holds, then the following lemma suffices to efficiently refute the $3 \mathrm{CNF}$ :

Lemma 4. Let $c$ be constant and $K$ be an unsatisfiable $3 C N F$ formula in the $X$ variables (where $n=|X|$ ). Assume that there is a set $S \subseteq X$ of at most $3(c-1)$ variables that hit all the clauses in $K$. Then there is a polynomial-size (in $n$ ) resolution refutation of $K$.

\footnotetext{
${ }^{6}$ A set of variables $S$ that "hit all the clauses in a CNF formula $K$ " is a set of variables for which every clause in $K$ contains some variable from $S$.
} 
Proof sketch: We simply run through all truth assignments to the variables in $S$ (since $|S| \leq 3(c-1)$, there are only constant number of such truth assignments). Under each truth assignment to the $S$ variables, $K$ becomes an unsatisfiable $2 \mathrm{CNF}$. It is known that any unsatisfiable $2 \mathrm{CNF}$ has a polynomial-size resolution refutation (cf. [Coo71]). Thus, we can refute $K$ with a polynomial-size resolution refutation.

If case (ii) in Lemma 3 holds, then it suffices to show that resolution under a big promise can efficiently refute any $3 \mathrm{CNF}$ formula $T$ with a constant number of clauses (for a sufficiently large constant), where each variable in $T$ occurs only once (such a $T$ is of course satisfiable, but it has less than an $\varepsilon$ fraction of satisfying assignments for a sufficiently large number of clauses). This is established in the following lemma.

Lemma 5. Fix the constant $c=3\left\lceil\log _{7 / 8}(\varepsilon / 2)\right\rceil$. Let $\Lambda=\varepsilon \cdot 2^{n}$, where $0<\varepsilon<1$ is a constant and $n$ is sufficiently large. Assume that $T$ is a $3 C N F$ with $c / 3$ clauses (and $c$ variables) over the $X$ variables, where each variable in $T$ occurs only once inside $T$. Then there is a polynomial-size resolution refutation of $T$ under the promise $\Lambda$.

Proof. The proof consists of constructing a sequence of polynomial-size circuits $C$ (where the parameters of the circuits in $C$ are taken from Definition 3.3; that is, $r=\lceil\log (1 / \varepsilon)\rceil$ and $t=2^{r}-1$ ), such that: (i) resolution can efficiently prove the injectivity and the pairwise disjointness of the images of the circuits in $C$; and (ii) there is a polynomial-size refutation of $T$ and $\mathrm{PRM}_{\Lambda, C}$. In other words, there is a polynomial-size derivation of the empty clause from the clauses of both $T$ and $\mathrm{PRM}_{\Lambda, C}$.

Without loss of generality we assume that the variables in $T$ are $x_{1}, \ldots, x_{c}$. The sequence $C$ consists of the circuits $C^{(1)}, \ldots, C^{(t)}$, where each circuit $C^{(i)}$ has $n-r$ input bits and $n$ output bits. Denote the Boolean circuit that computes the $j$ th output bit of $C^{(i)}$ by $C_{j}^{(i)}$ and let the input variables of all the circuits in $C$ be $\bar{W}:=\left\{w_{1}, \ldots, w_{n-r}\right\}$. As shown in equation (1), since the circuits in $C$ are intended to compute injective and pairwise image-disjoint maps, the image of $C$ would be of size $2^{n}-2^{n-r}$. We now define the map that each circuit in $C$ computes.

First, we determine the Boolean functions computed by the output bits in positions $c+1, \ldots, n$ in all the circuits in $C$. For all $1 \leq i \leq t$ and all $c+1 \leq j \leq n$ let $C_{j}^{(i)}(\bar{W})$ compute the $(j-r)$ th input variable $w_{j-r}$.

Second, we need to determine the rest of the output bits for all the circuits in $C$, that is, we need to determine the Boolean functions computed by $C_{j}^{(i)}$, for all $1 \leq i \leq t$ and all $1 \leq j \leq c$. Our intention is that for all $1 \leq i \leq t$, the (single output) circuits $C_{1}^{(i)}, \ldots, C_{c}^{(i)}$ should compute (when combined together) a Boolean map, denoted by $f_{i}$, from $c-r$ input bits $\bar{W}_{0}:=\left\{w_{1}, \ldots, w_{c-r}\right\}$, to $c$ output bits. The $j$ th output bit of $f_{i}$ (which is computed by $C_{j}^{(i)}$ ) is denoted by $f_{i, j}$, for $1 \leq j \leq c$. In other words, 
$f_{i}\left(\bar{W}_{0}\right)=f_{i, 1}\left(\bar{W}_{0}\right) \circ \cdots \circ f_{i, c}\left(\bar{W}_{0}\right)$, where $\circ$ denotes concatenation of bits (we shall describe the functions $f_{i}$ below). Summing it up for now, we have the following:

$$
\begin{gathered}
C_{1}^{(1)}\left(\bar{W}_{0}\right)=f_{1,1}\left(\bar{W}_{0}\right), \ldots, C_{c}^{(1)}\left(\bar{W}_{0}\right)=f_{1, c}\left(\bar{W}_{0}\right) \\
C_{c+1}^{(1)}\left(w_{c-r+1}\right)=w_{c-r+1}, \ldots, C_{n}^{(1)}\left(w_{n-r}\right)=w_{n-r} \\
\vdots \\
\begin{array}{c}
C_{1}^{(t)}\left(\bar{W}_{0}\right)=f_{t, 1}\left(\bar{W}_{0}\right), \ldots, C_{c}^{(t)}\left(\bar{W}_{0}\right)=f_{t, c}\left(\bar{W}_{0}\right), \\
C_{c+1}^{(t)}\left(w_{c-r+1}\right)=w_{c-r+1}, \ldots, C_{n}^{(t)}\left(w_{n-r}\right)=w_{n-r}
\end{array}
\end{gathered}
$$

where $C_{j}^{(i)}\left(w_{k}\right)=w_{k}$ denotes the fact that $C_{j}^{(i)}$ outputs the (input) variable $w_{k}$ (in which case we assume that the circuit $C_{j}^{(i)}$ consists of only a single gate: the variable $\left.w_{k}\right)$; and where $C_{j}^{(i)}\left(\bar{W}_{0}\right)=f_{i, j}\left(\bar{W}_{0}\right)$ denotes the fact that $C_{j}^{(i)}$ computes the function $f_{i, j}$ in the $c-r$ input variables $\bar{W}_{0}$.

We now describe the requirements from the functions $f_{i, j}$. Specifically, let $B \subseteq\{0,1\}^{c}$ be the set of all falsifying assignments ${ }^{7}$ to $T$ and denote by $\operatorname{Im}\left(f_{i}\right)$ the image of $f_{i}$, for all $1 \leq i \leq t$. We need the $f_{i}$ 's functions to map every input (over $c-r$ input bits) to a truth assignment (over the $c$ variable $x_{1}, \ldots, x_{c}$ ) that falsifies $T$ (that is, a truth assignment from $B$ ). We also need the $f_{i}$ 's to be injective and have pairwise disjoint images by the requirements of the promise axiom (Definition 3.3). So that, overall, the Boolean maps computed by the circuits in $C$ would discard all the truth assignments that satisfy $T$. To prove the existence of such $f_{i}$ 's we need the following claim:

Claim 2. There exists a collection of Boolean functions $f_{i}$, where $1 \leq i \leq t$, for which the following three properties hold.

(1) $\cup_{i=1}^{t} \operatorname{Im}\left(f_{i}\right) \subseteq B$;

(2) All the $f_{i}$ 's are injective and have pairwise disjoint images;

(3) All the $f_{i}$ 's depend on a constant number of input variables: $w_{1}, \ldots, w_{c-r}$ (and hence, all the $f_{i, j}$ 's depend only on these variables).

Proof. Each $f_{i}$ should depend on $c-r$ variables and should be injective, and further, each pair of $f_{i}$ 's should have disjoint images; thus we have:

$$
\left|\bigcup_{i=1}^{t} \operatorname{Im}\left(f_{i}\right)\right|=t \cdot 2^{c-r}=\left(2^{r}-1\right) \cdot 2^{c-r}=2^{c} \cdot\left(1-2^{-r}\right) .
$$

Hence, to prove the existence of the collection of $f_{i}$ 's with the required three properties it suffices to show that $\left|\cup_{i=1}^{t} \operatorname{Im}\left(f_{i}\right)\right| \leq|B|$, and by (4) it suffices to show:

\footnotetext{
${ }^{7}$ Note that the assignments here are actually partial truth assignments with respect to $X$, that is, they give truth values only to the variables $x_{1}, \ldots, x_{c}$ (these are all the variables in $T$ ).
} 


$$
2^{c} \cdot\left(1-2^{-r}\right) \leq|B| .
$$

Observe that the fraction of distinct assignments that satisfy $T$ is equal to the probability (over all truth assignments to $T$ ) that a uniformly chosen random truth assignment satisfies all the $c / 3$ clauses in $T$, which is equal to

$$
\left(\frac{7}{8}\right)^{c / 3}=\left(\frac{7}{8}\right)^{\left\lceil\log _{7 / 8}(\varepsilon / 2)\right\rceil}
$$

and so

$$
|B|=2^{c} \cdot\left(1-\left(\frac{7}{8}\right)^{\left\lceil\log _{7 / 8}(\varepsilon / 2)\right\rceil}\right) .
$$

Therefore, for (5) to hold it remains to show

$$
2^{-r} \geq\left(\frac{7}{8}\right)^{\left\lceil\log _{7 / 8}(\varepsilon / 2)\right\rceil},
$$

which holds because

$$
\begin{aligned}
& 2^{-r}=2^{-\lceil\log (1 / \varepsilon)\rceil} \geq 2^{-\log (1 / \varepsilon)-1}=2^{-\log (1 / \varepsilon)} / 2 \\
& \quad=\varepsilon / 2=\left(\frac{7}{8}\right)^{\log _{7 / 8}(\varepsilon / 2)} \geq\left(\frac{7}{8}\right)^{\left\lceil\log _{7 / 8}(\varepsilon / 2)\right\rceil} .
\end{aligned}
$$

Having established the existence of functions $f_{i}$ for which the three conditions in Claim 2 hold, we define each circuit $C_{j}^{(i)}$, for $1 \leq i \leq t$ and $1 \leq j \leq c$, to compute the function $f_{i, j}$. Since the domain of each $f_{i, j}$ is constant (that is, $\left.2^{c-r}\right)$ each $C_{j}^{(i)}$ can be of constant size.

Note that the circuits in $C=\left(C^{(1)}, \ldots, C^{(t)}\right)$ indeed compute $t$ injective Boolean maps that have pairwise disjoint images. Disjointness of images stems from the fact that the $f_{i}$ 's functions all have disjoint images, and injectivity stems from the fact that the $f_{i}$ 's are all injective, and that for each $1 \leq i \leq t$, the Boolean map computed by $C_{c+1}^{(i)} \circ \ldots \circ C_{n}^{(i)}$ (again, o denotes concatenation of bits) is exactly the identity map id: $\{0,1\}^{n-c} \rightarrow\{0,1\}^{n-c}$.

To complete the proof of Lemma 5 , we need to show that resolution can efficiently prove that indeed the circuits in $C$ all compute injective Boolean maps and have pairwise disjoint images (as well as to efficiently refute $T$ when assuming that $X$ can take only assignments from the image of $C$ ). This is done in the following claim:

Claim 3. Let $C$ be the sequence of circuits as devised above, and let $\mathrm{PRM}_{C, \Lambda}$ be the corresponding (big) promise axiom. Then there is a polynomial-size resolution refutation of $T$ and $\mathrm{PRM}_{C, \Lambda}$.

Proof. The proof follows by considering the encoding of the (big) promise axiom $\operatorname{PRM}_{C, \Lambda}$ via the encoding scheme in the Appendix (Section A.1) and showing how resolution can prove the empty clause from $T$ and this 
encoding. Here we shall use a less formal description; more details can be found in the appendix.

First we need resolution to prove the premise of the main implication in $\mathrm{PRM}_{C, \Lambda}$. This breaks into two parts corresponding to $\wedge_{i=1}^{t}\left(C^{(i)}\left(\overline{W_{1}}\right) \equiv C^{(i)}\left(\overline{W_{2}}\right) \rightarrow \overline{W_{1}} \equiv \overline{W_{2}}\right) \quad$ and $\quad \wedge_{1 \leq i<j \leq t} C^{(i)}\left(\overline{W_{1}}\right) \quad \not \equiv$ $C^{(j)}\left(\overline{W_{2}}\right)$.

For the first part, we need to refute the statement expressing that $C$ contains some circuit $C^{(i)}$ that computes a non-injective Boolean map. This can be efficiently refuted in resolution: Assume (inside resolution) that $C^{(i)}\left(\bar{W}_{1}\right) \equiv C^{(i)}\left(\bar{W}_{2}\right)$, for some $1 \leq i \leq t$, then by (3) we can efficiently prove (inside resolution) that for all $c-r+1 \leq j \leq n-r$ it happens that $w_{j}^{(1)} \equiv w_{j}^{(2)}$ (where $w_{j}^{(1)}$ is the $j$ th variable in $\bar{W}_{1}$, and $w_{j}^{(2)}$ is the $j$ th variable in $\bar{W}_{2}$ ) (see details in the appendix, and in particular Section A.1.3). Thus, it remains to refute the statement that for some $1 \leq j \leq c-r$ it happens that $w_{j}^{(1)} \not \equiv w_{j}^{(2)}$. This is indeed a contradiction by definition of the circuits in $C$ (as they compute injective maps). Since all the output bits $w_{j}^{(1)}, w_{j}^{(2)}$ for $1 \leq j \leq c-r$, are computed by constant size circuits $C_{j}^{(i)}$ for $1 \leq j \leq c-r$ and $1 \leq i \leq t$ (with constant number of input variables), such a contradiction can be refuted in constant size resolution refutation (again, see more details in the appendix).

The disjointness of the images of the (maps computed by the) circuits in $C$ is also efficiently provable inside resolution in a similar manner, and we shall not describe it here.

Therefore, we arrive (inside resolution) at the consequence of the main implication in the promise axiom: $\vee_{i=1}^{t} C^{(i)}\left(\bar{W}_{1}\right) \equiv X$. It remains to refute $T$ and $\vee_{i=1}^{t} C^{(i)}\left(\bar{W}_{1}\right) \equiv X$.

Again, $T$ has a constant number of variables (that is, $c$ ). Consider only the circuits that output to the variables $x_{1}, \ldots, x_{c}$ in $\vee_{i=1}^{t} C^{(i)}\left(\bar{W}_{1}\right) \equiv X$ : these are the circuits $C_{1}^{(i)}, \ldots, C_{c}^{(i)}$ for all $1 \leq i \leq t$. We shall denote the set of these circuits by $C^{\prime}$. The (functions computed by) the circuits in $C^{\prime}$ depend on a constant number of variable $\bar{W}_{0}$ and they have constant size. Denote by $Z$ the subformula of $\mathrm{PRM}_{C, \Lambda}$ that contains the (encoding of) the circuits in $C^{\prime}$ including the encoding of the statement that for some $1 \leq i \leq t$ the variables $x_{1}, \ldots, x_{c}$ are equal to the output of the circuits $C_{1}^{(i)}, \ldots, C_{c}^{(i)}$. By the definition of the circuits in $C$ (see condition (1) in Claim 2) $Z$ discards all the satisfying assignments of $T$ (over the $c$ variables in $T)^{8}$. Thus, $T$ and $Z$ constitute together a contradiction of constant size (as there are no satisfying assignments for both $T$ and $Z$ ). Therefore, there is a constant size resolution refutation of $T$ and $Z$.

\footnotetext{
${ }^{8}$ We have abused notation here, as we defined assignment discarding of only complete assignments to $X$ while here we say that $Z$ discards a partial assignment to the variables $x_{1}, \ldots, x_{c}$ only; but the definition of such partial assignment discarding is similar (consider the variables $x_{1}, \ldots, x_{c}$ to be the only original variables).
} 
This concludes the proof of Lemma 5 .

\section{Smaller Promise: Lower Bound}

In this section, we prove an exponential lower bound on the size of resolution refutations under the promise $2^{\delta n}$, for any constant $0 \leq \delta \leq 1$. The lower bound apply to random 3CNF formulas with $o\left(n^{3 / 2}\right)$ number of clauses (where $n$ is the number of variables in the $3 \mathrm{CNF}$ ). This lower bound matches the known lower bound on resolution refutation-size for random $3 \mathrm{CNF}$ formula (without any promise). Basically, the proof strategy of our lower bound is similar to that of Ben-Sasson and Wigderson [BSW01], except that we need to take care that every step in the proof works with the augmented (smaller) promise axiom.

The lower bound is somewhat stronger than described above in two respects. First, we show that restricting the set of all truth assignments $2^{n}$ to any smaller set (that is, not just those sets defined by small circuits) that consists of $2^{n}-2^{\delta n}$ assignments (for any constant $0 \leq \delta \leq 1$ ), does not give resolution any advantage in the average-case. One can think of such a restriction as modifying the semantic implication relation $\models$ to take into account only assignments from some prescribed set of assignments $S$, such that $|S|=2^{n}-2^{\delta n}$ (in other words, for two formulas $A, B$, we have that $A \models B$ under the restriction to $S$ iff any truth assignment from $S$ that satisfies $A$ also satisfies $B$ ). Formally, this means that the lower bound does not use the fact that the restricted domain of size $2^{n}-2^{\delta n}$ is defined by a sequence $C$ of polynomial-size circuits (nor the fact that the circuits in $C$ ought to have polynomial-size resolution proofs of their injectivity and pairwise disjointness).

Second, we could allow for a promise that is bigger than $2^{\delta n}$, and in particular for a promise of $2^{n\left(1-1 / n^{1-\xi}\right)}=2^{n} / 2^{n^{\xi}}$, for some constant $0<\xi<$ 1 (see Remark 1 below). The actual proof of the lower bound uses the smaller promise of $2^{\delta n}$, but the proof for a $2^{n} / 2^{n^{\xi}}$ promise is the same. (Although we have not defined precisely how the promise axioms are formulated in the case of a promise equal to $2^{n} / 2^{n^{\xi}}$, it is possible to formulate such promise axioms along the same lines described in Definition 3.4.)

The following defines the usual average-case setting of $3 \mathrm{CNF}$ formulas (there are other definitions, that are essentially similar):

Definition 5.1 (Random 3CNF formulas). For a $3 \mathrm{CNF}$ formula $K$ with $n$ variables $X$ and $\beta \cdot n$ clauses, we say that $\beta$ is the density of $K$. A random $3 \mathrm{CNF}$ formula on $n$ variables and density $\beta$ is defined by picking $\beta \cdot n$ clauses from the set of all $2^{3} \cdot\left(\begin{array}{l}n \\ 3\end{array}\right)$ clauses, independently and indistinguishably distributed, with repetitions. 
We say that an event (usually a property of a $3 \mathrm{CNF}$ in $n$ variables and density $\beta$ ) happens with high probability if it happens with $1-o(n)$ probability in the specified probability space (usually random $3 \mathrm{CNF}$ formulas as defined in Definition 5.1).

Our goal is to prove a lower bound on the average-case refutation-size of $3 \mathrm{CNF}$ formulas taken from the set of $3 \mathrm{CNF}$ formulas under a promise as defined in Definition 3.1 (note that the probability space defined in Definition 5.1 is defined over a different set of $3 \mathrm{CNF}$, that is, the set of all $3 \mathrm{CNF}$ formulas). For this purpose, we define a probability space over the set of $3 \mathrm{CNF}$ formulas under a promise: The distribution of random 3CNF formulas under a promise $\Lambda$ on $n$ variables and density $\beta$ is the distribution of random 3CNF formulas in Definition 5.1 conditioned on the event that the $3 \mathrm{CNF}$ is either unsatisfiable or has more than $\Lambda(n)$ satisfying assignments.

We now argue that to satisfy our goal to prove a lower bound on the average-case proof complexity of $3 \mathrm{CNF}$ formulas under a promise, it is sufficient to prove the lower bound result considering the distribution of random $3 \mathrm{CNF}$ formulas as defined in Definition 5.1.

It is well known that almost all $3 \mathrm{CNF}$ formulas with a density $\beta$ above a certain constant threshold (say, 5) are unsatisfiable. This means that any property of a $3 \mathrm{CNF}$ (with density above the threshold) that happens with high probability in the distribution in Definition 5.1 also happens with high probability in the distribution of random 3CNF formulas under a promise $\Lambda(n)$ (as defined above); this is because there are only a fraction $o(1)$ of $3 \mathrm{CNF}$ formulas (with a given fixed number of variables $n$ and a given fixed density $\beta$ above the threshold) that are satisfiable (and moreover have at least one satisfying assignment but less than $\Lambda(n)$ satisfying assignments). Thus, if we prove that with high probability a random 3CNF formula has no small promise resolution refutation then it implies also that with high probability a random $3 C N F$ formula under a promise has no small promise resolution refutation. Therefore, we shall consider from now on only the distribution of 3CNF formulas as defined in Definition 5.1, and forget about the other distribution.

5.1. The Lower Bound. Throughout this section we fix $0<\delta<1$ and $\Lambda=2^{\delta n}$. We also fix an arbitrary instance of a promise axiom $\mathrm{PRM}_{C, \Lambda}$ (from Definition 3.4; where $C$ is a sequence of the appropriate number of circuits, and each circuit in $C$ have the appropriate number of input and output bits). For $K$ a CNF formula, we denote by $\operatorname{Vars}(K)$ the set of variables that occur in $K$.

The following is the main theorem of this section. The lower bound matches that appearing in [BSW01] for resolution.

Theorem 6. Let $0<\delta<1$ and $0<\epsilon<1 / 2$. With high probability a random $3 C N F$ formula with $\beta=n^{1 / 2-\epsilon}$ requires a size $\exp \left(\Omega\left(\beta^{-4 /(1-\epsilon)} \cdot n\right)\right)$ resolution refutation under the promise $\Lambda=2^{\delta n}$. 
Remark 1. As mentioned above, we could allow in Theorem 6 for a promise that is bigger than $2^{\delta n}$, and precisely for a promise of $2^{n\left(1-\frac{1}{n^{1-\xi}}\right)}=2^{n} / 2^{n^{\xi}}$, for any constant $\xi$ such that $\frac{\epsilon}{(1-\epsilon)}<\xi<1$ (for instance, this allows for a promise of $\left.2^{n} / 2^{n^{1 / 3}}\right)$.

The proof strategy of Theorem 6 is to show that with high probability for a random $3 \mathrm{CNF}$ formula $K$ with density $\beta=n^{1 / 2-\epsilon}$, a resolution refutation under the promise $2^{\delta n}$ of $K$ must contain some clause $D$ of large width. Then we can apply the size-width tradeoff from Theorem 1 to reach the appropriate size lower bound.

However, we need to be a bit careful here, as in order to illustrate an exponential lower bound via the size-width tradeoff of Theorem 1, we need to guarantee that all the initial clauses (that is, all the axiom clauses) are of constant width. The $3 \mathrm{CNF}$ formula $K$ is certainly of constant width, but the clauses pertaining to the promise axiom $\mathrm{PRM}_{C, \Lambda}$ might not be (see the appendix for a detailed specification of these clauses). We can solve this problem easily: First, we add yet more extension variables to encode the clauses of the promise axiom with new constant width clauses. Second, we note that the original clauses of the promise axiom can be derived by a linear-size resolution proof from the new constant width version of the promise axiom (therefore, if there is a polynomial-size resolution refutation of $K$ using the original promise axiom, then there is also a polynomialsize resolution refutation of $K$ using the new constant width version of the promise axiom). Finally, we prove the exponential lower bound on resolution augmented with the constant width version of the promise axiom (instead of the original clauses pertaining to the promise axiom).

Let us explain now how to get the new constant-width promise axiom from the clauses pertaining to the original promise axiom from Definition 3.4 (as depicted in the appendix). Let $E=\ell_{1} \vee \ldots \vee \ell_{m}$ be a clause in the promise axiom that has more than constant width (that is, $\ell_{i}$ 's are literals and $m=\omega(1))$. Then, we replace the clause $E$ with the following collection of clauses:

$$
\ell_{1} \vee e_{1}, \neg e_{1} \vee \ell_{2} \vee e_{2}, \neg e_{2} \vee \ell_{3} \vee e_{3}, \ldots, \neg e_{m-1} \vee \ell_{m},
$$

where the $e_{i}$ 's are new extension variables. By resolving on the $e_{i}$ variables, one after the other, it is possible to derive with a linear-size resolution proof the original clause $E$ from the clauses in (7) (consider the first two clauses (from left) in (7), and resolve over the variable $e_{1}$, then the resolvent of this step is resolved over the variable $e_{2}$ with the third clause in (7), and so forth). (Note that every truth assignment that satisfies (7) also satisfies $E$, and so any clause that is semantically implied by $E$ (see the preliminaries, Section 2 ) is also semantically implied by (7). This means that the new constant width version of the promise axiom discards the same truth assignments to the variables $X$ as the original version of the promise axiom.) 
Thus, from now on in this section we assume that the promise axiom consists of clauses of a constant width.

The rest of this section is devoted to the proof of Theorem 6 .

For a clause $D$ define:

$$
\eta(D):=\min \left\{\left|K^{\prime}\right| \mid K^{\prime} \subseteq K \text { and }\left(\mathrm{PRM}_{C, \Lambda} \cup K^{\prime}\right) \models D\right\} .
$$

Remark 2. We use the symbol $\eta$ to distinguish it from a similar measure $\mu$ used in [BSW01]: Here we require the minimal set of clauses from $K$ that combined with the axiom $\mathrm{PRM}_{C, \Lambda}$ semantically imply $D$.

We show that with high probability for a random 3CNF formula with density $\beta=n^{1 / 2-\epsilon}$, for $0<\epsilon<1 / 2$, the following is true:

(1) Let $k=2 n \cdot(80 \beta)^{-2 /(1-\epsilon)}$. Then $\eta(\square) \geq k$.

(2) Any refutation of $K$ must contain a clause $D$ such that $k / 2 \leq \eta(D) \leq$ $k$.

(3) Any clause $D$ from 2 must have large width, and specifically $|D| \geq$ $\epsilon n(80 \beta)^{-2 /(1-\epsilon)}$ (which, by Theorem 1, concludes the proof).

The following two definitions are similar to those in [BSW01] (we refer directly to $3 \mathrm{CNF}$ formulas instead of 3-uniform hypergraphs):

Definition 5.2 (CNF Expansion). For a 3CNF formula $K$ with density $\beta=n^{1 / 2-\epsilon}$, for $0<\epsilon<1 / 2$, the expansion of $K$ is

$$
e(K):=\min \left\{2\left|\operatorname{Vars}\left(K^{\prime}\right)\right|-3\left|K^{\prime}\right| \mid \begin{array}{l}
K^{\prime} \subset K \text { and } \\
n \cdot(80 \beta)^{-2 /(1-\epsilon) \leq} \leq\left|K^{\prime}\right| \\
\leq 2 n \cdot(80 \beta)^{-2 /(1-\epsilon)}
\end{array}\right\} .
$$

Definition 5.3 (Partial matchability). A 3CNF formula $K$ with density $\beta=n^{1 / 2-\epsilon}$, for $0<\epsilon<1 / 2$, is called partially matchable if for all $K^{\prime} \subset K$ such that $\left|K^{\prime}\right| \leq 2 n \cdot(80 \beta)^{-2 /(1-\epsilon)}$ we have $\left|\operatorname{Vars}\left(K^{\prime}\right)\right| \geq\left|K^{\prime}\right|$.

The next lemma gives two properties of random 3CNF formulas that occur with high probability (see the appendix of [BSW01] for a proof). We then use this lemma to show that with high probability 1,2,3 above hold.

Lemma 7 ([BKPS02]). Let $0<\epsilon<1 / 2$ and let $K$ be a random 3CNF with $n$ variables and density $\beta=n^{1 / 2-\epsilon}$, then with high probability:

(1) $e(K) \geq \epsilon n(80 \beta)^{-2 /(1-\epsilon)}$; and

(2) $K$ is partially matchable.

We now prove 1 . In light of part (2) in Lemma 7, in order to prove that with high probability 1 holds it is sufficient to prove the following:

Lemma 8. Let $K$ be a $3 C N F$ formula in the $X$ variables with density $\beta=$ $n^{1 / 2-\epsilon}$, for $0<\epsilon<1 / 2$. If $K$ is partially matchable then $\eta(\square) \geq 2 n$. $(80 \beta)^{-2 /(1-\epsilon)}$. 
Proof. By partial matchability of $K$, for all $K^{\prime} \subset K$ such that $\left|K^{\prime}\right| \leq 2 n$. $(80 \beta)^{-2 /(1-\epsilon)}$ it happens that $\left|\operatorname{Vars}\left(K^{\prime}\right)\right| \geq\left|K^{\prime}\right|$. Thus, by Hall's Theorem we can choose a distinct variable (representative) from each clause in $K^{\prime}$ and set it to satisfy its clause. Clearly, $\left|\operatorname{Vars}\left(K^{\prime}\right)\right| \leq 3\left|K^{\prime}\right| \leq 6 n \cdot(80 \beta)^{-2 /(1-\epsilon)}$, and so there is a (partial) truth assignment $\rho$ to at most $6 n \cdot(80 \beta)^{-2 /(1-\epsilon)}$ variables in $X$ that satisfies $K^{\prime}$. Since $\beta=n^{1 / 2-\epsilon}$,

$$
6 n \cdot(80 \beta)^{-2 /(1-\epsilon)}=6 \cdot 80^{-2 /(1-\epsilon)} \cdot n^{\epsilon /(1-\epsilon),}
$$

which, by $0<\epsilon<1 / 2$, is equal to $O\left(n^{\lambda}\right)$ for some $0<\lambda<1$. Thus for sufficiently large $n$ there are more than $\delta n$ variables from $X$ not set by $\rho$, which means that there are more than $2^{\delta n}$ different ways to extend $\rho$ into truth assignments (to all the variables in $X$ ) that satisfy $K^{\prime}{ }^{9}$ Since the promise axiom $\mathrm{PRM}_{C, \Lambda}$ can discard up to $2^{\delta n}$ truth assignments to the $X$ variables, we get that $\mathrm{PRM}_{C, \Lambda} \cup K^{\prime}$ is satisfiable (any assignment to $X$ that is not discarded by $\mathrm{PRM}_{C, \Lambda}$ can be extended to the extension variables in a way that satisfies $\mathrm{PRM}_{C, \Lambda}$ ).

We have thus showed that every collection $K^{\prime}$ containing at most $2 n \cdot(80 \beta)^{-2 /(1-\epsilon)}$ clauses from $K$ and augmented with the promise axiom $\mathrm{PRM}_{C, \Lambda}$ is satisfiable. This implies in particular that $\eta(\square) \geq 2 n$. $(80 \beta)^{-2 /(1-\epsilon)}$.

We now prove 2 . Note that the resolution rule is sub-additive with respect to $\eta$ in the sense that for all three clauses $E, F, D$ such that $D$ is a resolvent of $E$ and $F$, it holds that

$$
\eta(E)+\eta(F) \geq \eta(D) .
$$

We also clearly have that for every axiom clause $E$ (either from $K$ or from the promise axiom):

$$
\eta(E)=1
$$

Let

$$
k=2 n \cdot(80 \beta)^{-2 /(1-\epsilon)} .
$$

By Lemma 8, with high probability for a 3CNF formula $K$ with density $\beta=n^{1 / 2-\epsilon}$ (for $0<\epsilon<1 / 2$ ) it happens that $\eta(\square) \geq k$. By sub-additivity of the resolution rule with respect to $\eta$, in any resolution refutation of $K$ under the promise $\Lambda$, there ought to be some clause $D$ such that

$$
k / 2 \leq \eta(D) \leq k .
$$

We now prove 3 . Let $D$ be a clause such that $k / 2 \leq \eta(D) \leq k$ from 2 and let $K^{\prime}$ be the (minimal) set of clauses from $K$ for which $\operatorname{PRM}_{C, \Lambda} \cup K^{\prime} \models D$ and $k / 2 \leq\left|K^{\prime}\right| \leq k$. We shall prove that (with high probability for a

\footnotetext{
${ }^{9}$ Actually, for sufficiently large $n$ there are more than $\Omega\left(n-n^{\epsilon /(1-\epsilon)}\right)$ such variables, from which we can assume the bigger promise $\Lambda=2^{n} / 2^{n^{\xi}}$, for any $\frac{\epsilon}{(1-\epsilon)}<\xi<1$, as noted in Remark 1.
} 
random $3 \mathrm{CNF})|D| \geq \epsilon n(80 \beta)^{-2 /(1-\epsilon)}$. In light of Lemma 7 part (1), in order to prove this, it is sufficient to prove the next two lemmas.

Define $\partial K^{\prime}$, called the boundary of $K^{\prime}$, to be the set of variables in $K^{\prime}$ that occur only once in $K^{\prime}$ (in other words, each variable in $\partial K^{\prime}$ appears only in one clause in $K^{\prime}$ ).

Lemma 9. $\left|\partial K^{\prime}\right| \geq e(K)$.

Proof. Every variable not in $\partial K^{\prime}$ must be covered by at least two distinct clauses in $K^{\prime}$, and so $\left|\operatorname{Vars}\left(K^{\prime}\right)\right| \leq\left|\partial K^{\prime}\right|+\frac{1}{2} \cdot\left(3\left|K^{\prime}\right|-\left|\partial K^{\prime}\right|\right)$. Thus, we have $\left|\partial K^{\prime}\right| \geq 2\left|\operatorname{Vars}\left(K^{\prime}\right)\right|-3\left|K^{\prime}\right| \geq e(K)$ (where the last inequality is by Definition 5.2 and since $\left.k / 2 \leq\left|K^{\prime}\right| \leq k\right)$.

Lemma 10. $|D| \geq\left|\partial K^{\prime}\right|$.

Proof. Let $x_{i} \in \partial K^{\prime}$, for some $1 \leq i \leq n$, and denote by $K_{i}$ the (unique) clause from $K^{\prime}$ that contains $x_{i}$. Assume by a way of contradiction that $x_{i}$ does not occur in $D$.

By minimality of $K^{\prime}$ with respect to $\eta$ and $D$ there exists an assignment $\alpha$ (here we treat $\alpha$ as a total truth assignment, that is, a truth assignment to both the $X$ variables and the extension variables in the promise axiom) such that

$$
\left(K^{\prime} \backslash K_{i}\right)(\alpha)=1 \text { and } D(\alpha)=0
$$

(as otherwise $\left(K^{\prime} \backslash K_{i}\right) \models D$ which clearly implies $\operatorname{PRM}_{C, \Lambda} \cup\left(K^{\prime} \backslash K_{i}\right) \models D$, which then contradicts the minimality of $K^{\prime}$ with respect to $\eta$ and $\left.D\right)$.

By assumption, $x_{i}$ occurs neither in $K^{\prime} \backslash K_{i}$ nor in $D$. Hence, we can flip the value of $\alpha$ on $x_{i}$ so that $K_{i}(\alpha)=1$ while still keeping (9) true. We thus have:

$$
K^{\prime}(\alpha)=1 \text { and } D(\alpha)=0
$$

Since $\left|K^{\prime}\right| \leq k$, we have that $\left|\operatorname{Vars}\left(K^{\prime}\right)\right| \leq 3 k=6 n \cdot(80 \beta)^{-2 /(1-\epsilon)}$ (recall that $\left|K^{\prime}\right|$ is the number of clauses in $\left.K^{\prime}\right)$. If $|D| \geq\left|\partial K^{\prime}\right|$ we are done. Otherwise,

$$
\left|\operatorname{Vars}\left(K^{\prime}\right)\right|+|D|<\left|\operatorname{Vars}\left(K^{\prime}\right)\right|+\left|\partial K^{\prime}\right| \leq 2\left|\operatorname{Vars}\left(K^{\prime}\right)\right| \leq 12 n \cdot(80 \beta)^{-2 /(1-\epsilon)} .
$$

Thus, similar to equation (8), for sufficiently large $n$, the total number of distinct variables in $K^{\prime}$ and $D$ is at most $\left|\operatorname{Vars}\left(K^{\prime}\right)\right|+|D|=O\left(n^{\lambda}\right)$, for some $0<\lambda<1$. This means that for sufficiently large $n$ there are more than $\delta n$ variables from $X$ for which flipping the value of $\alpha$ on them still validates (10). ${ }^{10}$ Hence, there are more than $2^{\delta n}$ distinct assignments to the $X$ variables for which (10) holds.

The promise axiom $\mathrm{PRM}_{C, \Lambda}$ discards at most $2^{\delta n}$ assignments to the $X$ variables. This means that there are at most $2^{\delta n}$ assignments $\rho$ to the $X$ variables that falsify $\mathrm{PRM}_{C, \Lambda}$ (that is, that every extension of $\rho$ to all the

\footnotetext{
${ }^{10}$ Again, similar to what was noted in the proof of Lemma 8 , for sufficiently large $n$ there are actually more than $\Omega\left(n-n^{\epsilon /(1-\epsilon)}\right)$ such variables.
} 
extension variables falsifies $\mathrm{PRM}_{C, \Lambda}$, while all other assignments $\rho$ to the $X$ variables have an extension (to all the the extension variables) that satisfies $\mathrm{PRM}_{C, \Lambda}$. Thus, by the previous paragraph there ought to be at least one assignment $\rho$ to the $X$ variables that has an extension $\rho^{\prime}$ to the extension variables, such that

$$
\operatorname{PRM}_{C, \Lambda}\left(\rho^{\prime}\right)=1, K^{\prime}\left(\rho^{\prime}\right)=1 \text { and } D\left(\rho^{\prime}\right)=0,
$$

which contradicts the assumption that $\operatorname{PRM}_{C, \Lambda} \cup K^{\prime} \models D$.

\section{Conclusion}

This paper establishes a new framework of propositional proof systems that are able to separate the unsatisfiable CNF formulas from the set of CNF formulas having many satisfying assignments. We were able to analyze the complexity of basic cases pertaining to such proof systems, such as the case of a big promise (a constant fraction of all truth assignments) and the average-case proof complexity of refutations under a smaller promise (that is, a promise of $2^{\delta n}$, for any constant $0<\delta<1$ ).

One question we have not addressed is what can be gained (if at all) when we augment a stronger proof system than resolution, like boundeddepth Frege proof system or Frege proof system, with the promise axioms (for a small promise like $2^{\delta n}$, as for a big promise already resolution can efficiently refute all unsatisfiable 3CNF formulas).

Another question that arises is whether the fact that we require the Boolean circuits in the promise axioms to be provably injective and to provably posses disjoint images (that is, provably inside resolution) constitutes a real restriction. (Note that the lower bound for resolution under the promise $2^{\delta n}$ in Section 5 did not use at all these requirements.) In other words, we ask whether there is a sequence of circuits $C^{(1)}, \ldots, C^{(t)}$ for which adding the axiom $\vee_{i=1}^{t} C^{(i)}(\bar{W}) \equiv X$ (where the parameter $t$ and the number of variables in $m$ are taken from the smaller promise axiom 3.4) to resolution (or a stronger proof system) gives a super-polynomial speed-up for some contradictory family of formulas over standard resolution (or the stronger proof system); but that we cannot prove efficiently in resolution (or the stronger proof system) that $C^{(1)}, \ldots, C^{(t)}$ are injective or that they have pairwise disjoint images?

A different and more general task is to come up with other natural models of propositional proof systems that capture a "relaxed" notion of soundness. For instance, Pitassi [Pit06] suggested considering "approximate proofs" in the framework of algebraic proof systems.

Finally, we have not dealt directly in this paper with the promise $\Lambda=$ $2^{n} / \operatorname{poly}(n)$, though it is most likely that a similar upper bound (with a similar proof) to that shown in Section 4 also holds for this promise (when the promise axiom is modified accordingly). In this respect it is worth mentioning that Krajíček [Kra07] observed that the work of Razborov and Rudich [RR97] implies the existence (under a cryptographic conjecture) of a 
Boolean function $g$ with $n^{\delta}$ input bits (denoted by $y_{1}, \ldots, y_{n^{\delta}}$ ) and $n$ output bits (denoted by $\left.g_{1}\left(y_{1}, \ldots, y_{n^{\delta}}\right), \ldots, g_{n}\left(y_{1}, \ldots, y_{n^{\delta}}\right)\right)$, for any constant $0<$ $\delta<1$, that has the following property: Given any CNF formula $K$ in $n$ variables $x_{1}, \ldots, x_{n}$, substituting $g_{1}\left(y_{1}, \ldots, y_{n^{\delta}}\right), \ldots, g_{n}\left(y_{1}, \ldots, y_{n^{\delta}}\right)$ for the original $x_{i}$ variables in $K$ yields a new CNF formula that is unsatisfiable only if $K$ has at most $2^{n} / n^{\omega(1)}$ satisfying assignments. This means that under the promise $2^{n} /$ poly $(n)$ the substitution $g$ is sound: Any unsatisfiable CNF formula (clearly) stays unsatisfiable after the substitution, while any CNF with more than $2^{n} / \operatorname{poly}(n)$ satisfying assignments stays satisfiable after the substitution.

\section{Appendix A. EnCODINGS}

A.1. Encoding of Boolean Circuits and Promise Axioms. In this section we describe in detail how the promise axiom (see Definition 3.3) is encoded as a CNF formula. As already mentioned in the Preliminaries (Section 2), by Cook's Theorem there is always an efficient way to encode a Boolean circuit as a CNF formula using extension variables (that is, a CNF with size $O(s \cdot \log (s))$ can encode a circuit of size $s)$. However, we shall need to be more specific regarding the actual way the encoding is done since we require that resolution should be able to efficiently prove some basic facts about the encoded circuits.

A.1.1. Boolean circuit encoding. The following definition is similar to the circuit encoding defined in Alekhnovich et al. in [ABSRW04] (note that it deals with a single output bit Boolean circuit):

Definition A.1 (Encoding of Boolean circuits). Let $C(\bar{W})$ be a Boolean circuit (with $\vee, \wedge$ as fan-in two gates and $\neg$ a fan-in one gate) and $m$ input variables $\bar{W}:=w_{1}, \ldots, w_{m}$ and a single output bit. For every gate $v$ of the circuit $C$ we introduce a special extension variable $y_{v}$. For input gates $w_{j}$ $(1 \leq j \leq m)$ we identify $y_{w_{j}}$ with $w_{j}$. We denote by $y^{1}$ the literal $y$ and by $y^{0}$ the literal $\neg y$. The CNF formula $\|C(\bar{W})\|$ consists of the following clauses:

(i) $y_{v_{1}}^{\bar{\epsilon}_{1}} \vee y_{v_{2}}^{\bar{\epsilon}_{2}} \vee y_{v}^{\pi_{\circ}\left(\epsilon_{1}, \epsilon_{2}\right)}$, where $v$ is a $\circ \in\{\vee, \wedge\}$ gate in $C$ and $v_{1}, v_{2}$ are the two input gates of $v$ in $C$ and $\left\langle\epsilon_{1}, \epsilon_{2}\right\rangle$ is any vector in $\{0,1\}^{2}$ and $\pi_{\circ}$ is the truth table function of $\circ($ and $\overline{0}=1, \overline{1}=0)$;

(ii) $y_{v_{1}}^{\bar{\epsilon}_{1}} \vee y_{v}^{\pi_{\neg}\left(\epsilon_{1}\right)}$, where $v$ is a $\neg$ gate in $C$ and $v_{1}$ is the single input gates of $v$ in $C$, and $\epsilon_{1} \in\{0,1\}$ and $\pi_{\neg}$ is the truth table function of $\neg$.

We write $\|C(\bar{W})\|(y)$ to indicate explicitly that the output gate $v$ of $C$ is encoded by the extension variable $y$.

A.1.2. Encoding of the promise axioms. We now give a rather detailed description of how the promise axioms are encoded as CNF formulas. We shall consider only the 'big' promise axiom (Definition 3.3), but the other variant (Definition 3.4) is similar. We encode the promise axioms in a bottom-up 
manner, encoding the sub-formulas separately, and then combining all of them together.

We assume that a Boolean circuit $C(\bar{W})$ with $n$ output bits is encoded as $n$ distinct circuits and we write $\|C(\bar{W})\|(\bar{Y})$ to indicate explicitly that the output gates $v_{1}, \ldots, v_{n}$ of $C$ are encoded by the extension variables $y_{1}, \ldots, y_{n}$ (where $\bar{Y}:=\left\{y_{1}, \ldots, y_{n}\right\}$ ). This means that $\|C(\bar{W})\|(\bar{Y})$ is the CNF formula $\wedge_{i=1}^{n}\left\|C_{i}(\bar{W})\right\|\left(y_{i}\right)$, where $C_{i}(\bar{W})$ is the circuit computing the $i$ th output bit of $C(\bar{W})$ and $y_{i}$ is the variable that encodes (see Definition A.1) the (single) output bit of $C_{i}(\bar{W})$. We also require that if the (function computed by the) circuit $C(\bar{W})$ does not depend, ${ }^{11}$ on some input bit $w_{i}$, then $w_{i}$ does not occur in the encoding of $C(\bar{W})$.

Let $1 \leq k \leq t$ (where the parameter $t$ is taken from Definition 3.3). We first encode as a CNF formula the negation of following sub-formula from the promise axiom:

$$
C^{(k)}\left(\bar{W}_{1}\right) \equiv C^{(k)}\left(\bar{W}_{2}\right) \rightarrow \bar{W}_{1} \equiv \bar{W}_{2} .
$$

We denote this CNF encoding by $\neg \mathrm{INJ}_{k}$ (where INJ stands for injective).

Definition A.2 $\left(\neg \mathrm{INJ}_{k}\right)$. Let $1 \leq k \leq t$ and $m=n-r$ (all the parameters are taken from Definition 3.3). Let $\bar{W}_{1}:=\left\{w_{1}^{(1)}, \ldots, w_{m}^{(1)}\right\}$, $\bar{W}_{2}:=\left\{w_{1}^{(2)}, \ldots, w_{m}^{(2)}\right\}, \bar{Y}_{k}:=\left\{y_{1}^{(k)}, \ldots, y_{n}^{(k)}\right\}$ and $Z_{k}:=\left\{z_{1}^{(k)}, \ldots, z_{n}^{(k)}\right\}$ be sets of new distinct extension variables. The CNF formula $\neg I N J_{k}$ consists of the following set of clauses:

(1) $\left\|C^{(k)}\left(\bar{W}_{1}\right)\right\|\left(\bar{Y}_{k}\right) ; \quad\left\|C^{(k)}\left(\bar{W}_{2}\right)\right\|\left(\bar{Z}_{k}\right)$ (expresses that $\bar{Y}_{k}, \bar{Z}_{k}$ are the output bits of $C^{(k)}\left(\bar{W}_{1}\right), C^{(k)}\left(\bar{W}_{2}\right)$, respectively);

(2) $\neg u_{i} \vee \neg y_{i}^{(k)} \vee z_{i}^{(k)} ; \neg u_{i} \vee y_{i}^{(k)} \vee \neg z_{i}^{(k)}$, for all $1 \leq i \leq n$ (expresses that $u_{i}$ implies $\left.y_{i}^{(k)} \equiv z_{i}^{(k)}\right)$;

(3) $v_{i} \vee w_{i}^{(1)} \vee w_{i}^{(2)} ; v_{i} \vee \neg w_{i}^{(1)} \vee \neg w_{i}^{(2)}$; for al $1 \leq i \leq m$ (expresses that $\neg v_{i}$ implies $\left.w_{i}^{(1)} \equiv w_{i}^{(2)}\right)$;

(4) $u_{1}, \ldots, u_{n}$ (expresses that $\bar{Y} \equiv \bar{Z}$ );

(5) $\neg v_{1} \vee \ldots \vee \neg v_{m}$ (expresses that $\bar{W}_{1} \not \equiv \bar{W}_{2}$ );

For simplicity of writing we introduce the following notation: Let $\ell$ be a literal and let $A$ be a CNF formula. We denote by $\ell \emptyset A$ the set of clauses (that is, the CNF formula) that results by adding to each clause of $A$ the literal $\ell$.

We now encode as a CNF formula denoted by $\neg$ INJ the negation of

$$
\bigwedge_{k=1}^{t}\left(C^{(k)}\left(\bar{W}_{1}\right) \equiv C^{(k)}\left(\bar{W}_{2}\right) \rightarrow \bar{W}_{1} \equiv \bar{W}_{2}\right) .
$$

Definition A.3 $(\neg \mathrm{INJ})$. The $C N F$ formula $\neg I N J$ consists of the following set of clauses:

\footnotetext{
${ }^{11}$ We say that a Boolean function $f$ does not depend on an input bit $w_{i}$ if for all input assignments $\alpha$ to $f$, flipping the truth value of $w_{i}$ in $\alpha$ does not change the value of $f$.
} 
(1) $\neg p_{k} \oslash \neg \mathrm{INJ}_{k}$ for al $1 \leq k \leq t$ (expresses that $\mathrm{INJ}_{k}$ implies $\neg p_{k}$ );

(2) $p_{1} \vee \ldots \vee p_{t}\left(\right.$ expresses $\vee_{k=1}^{t} \neg \mathrm{INJ}_{k}$.)

In a similar manner one can encode as a CNF the negation of the formula

$$
\bigwedge_{1 \leq i<j \leq t}\left(C^{(i)}\left(\bar{W}_{1}\right) \not \equiv C^{(j)}\left(\bar{W}_{2}\right)\right)
$$

denoted by $\neg$ DSJ (where DSJ stands for disjoint). We shall not develop the encoding precisely, as this is pretty much similar to $\neg$ INJ.

The last part of the promise axiom we need to encode is the formula

$$
\bigvee_{i=1}^{t} C^{(i)}\left(\bar{W}_{1}\right) \equiv X .
$$

We denote the CNF encoding of this formula by RST (which stands for restriction). Again, this is similar to the encoding of $\neg$ INJ, but we show how to encode it anyway, since we would like to illustrate in the sequel how resolution can use RST to efficiently prove some basic facts about the $X$ variables (in the case the circuits in $C$ have certain simple form).

Definition A.4 (RST). For every $1 \leq k \leq t$, recall that $\bar{Y}_{k}:=$ $\left\{y_{1}^{(k)}, \ldots, y_{n}^{(k)}\right\}$ are the output variables of $\left\|C^{(k)}\left(\bar{W}_{1}\right)\right\|$ from Definition A.2. The $C N F$ formula $R S T$ consists of the following set of clauses:

(1) $\neg f_{i}^{(k)} \vee \neg y_{i}^{(k)} \vee x_{i} ; \neg f_{i}^{(k)} \vee y_{i}^{(k)} \vee \neg x_{i}$ for all $1 \leq i \leq n$ (expresses that $f_{i}^{(k)}$ implies $\left.y_{i}^{(k)} \equiv x_{i}\right)$;

(2) $\neg h_{k} \vee f_{1}^{(k)}, \ldots, \neg h_{k} \vee f_{n}^{(k)}$ (expresses that $h_{k}$ implies $\left.\bar{Y}_{k} \equiv X\right)$;

(3) $h_{1} \vee \ldots \vee h_{t} \quad\left(\right.$ expresses $\bigvee_{i=1}^{t} \bar{Y}_{k} \equiv X$.)

Finally, the promise axiom $\mathrm{PRM}_{C, \Lambda}$ is the following CNF formula:

Definition A.5 ( $\mathrm{CNF}$ encoding of $\left.\mathrm{PRM}_{C, \Lambda}\right)$. The CNF encoding of the promise axiom $\mathrm{PRM}_{C, \Lambda}$ consists of the following clauses:

(1) $\neg q_{1} \oslash \neg$ INJ (expresses that INJ implies $\neg q_{1}$ );

(2) $\neg q_{2} \oslash \neg$ DSJ (expresses that DSJ implies $\neg q_{2}$ );

(3) $q_{1} \oslash\left(q_{2} \oslash \mathrm{RST}\right)$ (expresses $\neg \mathrm{INJ} \vee \neg \mathrm{DSJ} \vee \mathrm{RST}$, which is equivalent to $\mathrm{INJ} \wedge \mathrm{DSJ}) \rightarrow \mathrm{RST})$.

A.1.3. Proving basic facts about encoded circuits inside resolution. The following simple claim illustrates how one can reason inside resolution, and specifically can "eliminate implications" inside resolution. Consider, for instance, line 1 in $\mathrm{PRM}_{C, \Lambda}$ (Definition A.5). This line expresses that INJ implies $\neg q_{1}$. In other words, it is logically equivalent to INJ $\rightarrow \neg q_{1}$. Assume that we already know INJ (which formally means that we have a resolution refutation of $\neg$ INJ). We would like to arrive inside resolution at $\neg q_{1}$. The following straightforward claim illustrates how to do this in resolution. 
Claim 4. Let $A$ be an unsatisfiable CNF formula with a resolution refutation of size $s$ and let $\ell$ be any literal. Then there is a resolution proof of $\ell$ from $\ell \otimes A$ of size $s$.

Proof. Assume that the resolution refutation of $A$ is the sequence of clauses $A_{1}, \ldots, A_{s}$, where $A_{s}=\square$ (the empty clause). Then $\ell \vee A_{1}, \ldots, \ell \vee A_{s}$ is a resolution proof of $\ell \vee \square=\ell$ from $\ell \square A$ (we assume that $\ell$ is not in any $A_{i}$; or else, by the weakening rule, the claim also holds).

Note that Claim 4 implies that if there is a refutation of $\neg$ INJ of size $s$, then there is also a proof of $\neg q_{1}$ of the same size $s$, from line 1 in $\operatorname{PRM}_{C, \Lambda}$ (Definition A.5).

We now illustrate how resolution can efficiently prove a certain simple fact about simple circuits. This is needed (among other efficient proofs of similar simple facts) in order to show the upper bound in Section 4 (and specifically, it is used in Claim 3). Other similar facts about the Boolean circuits constructed in Section 4 can be proved inside resolution in a similar manner, and we shall not describe these proofs here.

For some $1 \leq k \leq t$, let $C^{(k)}$ be a circuit from a sequence of circuits $C$ (as in the promise axioms), where $m$ and $n$ are the number of input and output variables of $C^{(k)}$, respectively. Assume that the $i$ th output bit of $C^{(k)}$ computes the $j$ th input bit $w_{j}$ for some $1 \leq j \leq m$ and $1 \leq i \leq n$. We require that resolution can efficiently refute (the encoding via Definition A.2 of):

$$
C^{(k)}\left(\bar{W}_{1}\right) \equiv C^{(k)}\left(\bar{W}_{2}\right) \wedge w_{j}^{(1)} \not \equiv w_{j}^{(2)}
$$

(note that by assumption this is clearly a contradiction).

Since $C_{i}^{(k)}$ just computes the $j$ th input bit $w_{j}$, then in fact we can assume that the encoding $\left\|C_{i}^{(k)}(\bar{W})\right\|\left(y_{i}\right)$ consists of only the single clause $w_{j}$ (remember that by Definition A.1 we identify between the variable encoding an input gate with the input variable $w_{j}$ itself; and here we know that $w_{j}$ is also the output variable). Thus, by 2 in Definition A.2 we have that the output bit $y_{j}$ of $C_{i}^{(k)}\left(\bar{W}_{1}\right)$ equals the output bit $z_{j}$ of $C_{i}^{(k)}\left(\bar{W}_{2}\right)$, and $y_{j}$ is actually $w_{j}^{(1)}$ and $z_{j}$ is actually $w_{j}^{(2)}$. Therefore, by Definition A.2 3 , we can prove $v_{j}$. So, by one resolution rule applied to A.2 5 , we are left with $\vee_{i \neq j} v_{i}$.

Assume that all but a constant number of the output bits of $C^{(k)}$ compute some (distinct) input bit $w_{j}$, for some $1 \leq j \leq m$ (this assumption corresponds to the circuits we build in Section 4). Then the process described in the previous paragraphs can be iterated for all such output bits of $C^{(k)}$, in order to cut off (that is, resolve over) all the $v_{j}$ variables in clause 5 in Definition A.2, until we reach only a disjunction of constant number of variables $v_{j}$ instead of clause 5 in A.2.

We are thus left with a constant number of circuits depending only on a constant number of input variables. Therefore, we can now refute with a 
polynomial-size resolution refutation the encoding of

$$
C^{(k)}\left(\bar{W}_{1}\right) \equiv C^{(k)}\left(\bar{W}_{2}\right) \wedge \bar{W}_{1} \not \equiv \bar{W}_{2}
$$

(if indeed the circuit $C^{(k)}$ computes an injective map, which means that (12) is unsatisfiable).

A.1.4. Comments on decoding the encoded promise axioms. In order to assert that promise resolution is a Cook-Reckhow proof system (see the first paragraph in Section 1.1 for a definition) we need to make sure that a promise resolution refutation can be identified as such in polynomial-time. For this, one needs to be able to verify whether a given CNF is an instance of the promise axiom.

As mentioned in Section 3, this can be done by "decoding" the CNF that encodes the promise axiom $\mathrm{PRM}_{C, \Lambda}$ and then checking that each circuit in $C$ has the right number of input and output bits. Here we illustrate how this can be achieved.

First, it is possible to identify which are the clauses pertaining to the promise axioms out of all the clauses in the refutation (for instance, any clause used as an axiom that is not one of the clauses of the CNF meant to be refuted). Second, it is possible to identify which are the clauses of the promise axiom that are part of the circuit encoding (that is, clauses in line 1 in Definition A.2). It is then possible to decode the circuits from the encoding, and check that the circuits are legitimate ones and have the intended number of input and output variables (we omit the details).

\section{ACKNOWLEDGMENTS}

The second author is indebted to Ran Raz for very helpful conversations that led to the present paper. We wish to thank Jan Krajíček for commenting on an early version of this paper and Eli Ben-Sasson and Amnon Ta-Shma for useful correspondence and conversations.

\section{REFERENCES}

[ABSRW04] Michael Alekhnovich, Eli Ben-Sasson, Alexander A. Razborov, and Avi Wigderson. Pseudorandom generators in propositional proof complexity. SIAM J. Comput., 34(1):67-88, 2004. (A preliminary version appeared in Proceedings of the 41st Annual Symposium on Foundations of Computer Science (Redondo Beach, CA, 2000)). A.1.1

[BKPS02] Paul Beame, Richard Karp, Toniann Pitassi, and Michael Saks. The efficiency of resolution and Davis-Putnam procedures. SIAM J. Comput., 31(4):10481075, 2002. 1.1, 4, 7

[BS01] Eli Ben-Sasson. Expansion in Proof Complexity. PhD thesis, Hebrew University, Jerusalem, Israel, September 2001. 4

[BSW01] Eli Ben-Sasson and Avi Wigderson. Short proofs are narrow-resolution made simple. J. ACM, 48(2):149-169, 2001. (A preliminary version appeared in Proceedings of the 31th Annual ACM Symposium on the Theory of Computing (Atlanta, GA, 1999)). 1.1, 4, 1.2, 2.3, 1, 5, 5.1, 2, 5.1, 5.1 
[Coo71] Stephen A. Cook. The complexity of theorem proving procedures. In Proceedings of the 3rd Annual ACM Symposium on the Theory of Computing, pages 151-158. ACM, New York, 1971. 4, 4

[CR79] Stephen A. Cook and Robert A. Reckhow. The relative efficiency of propositional proof systems. The Journal of Symbolic Logic, 44(1):36-50, 1979. 1.1

[FKO06] Uriel Feige, Jeong Han Kim, and Eran Ofek. Witnesses for non-satisability of dense random $3 \mathrm{CNF}$ formulas. In Proceedings of the IEEE $4 \mathrm{7th}$ Annual Symposium on Foundations of Computer Science, 2006. 1.1

[Hir98] Edward Hirsch. A fast deterministic algorithm for formulas that have many satisfying assignments. Logic Journal of the IGPL, 6(1):5971, 1998. 1.1, 1

[Kra07] Jan Krajíček. Substitutions into propositional tautologies. Information Processing Letters, 101:163-167, 2007. 6

[Kra07] Jan Krajíček. Personal communication, 2007. 2

[Pit06] Toniann Pitassi. Using hardness in proof complexity. Talk given in New Directions in Proof Complexity, an Isaac Newton institute workshop, Cambridge, April 2006. 6

[Rob65] J. Alan Robinson. A machine-oriented logic based on the resolution principle. Journal of the ACM, 12(1):23-41, January 1965. 1.1

[RR97] Alexander A. Razborov and Steven Rudich. Natural proofs. J. Comput. System Sci., 55(1, part 1):24-35, 1997. (A Preliminary version appeared in Proceedings of the 26th Annual ACM Symposium on the Theory of Computing (Montreal, PQ, 1994)). 6

[Tre04] Luca Trevisan. A note on approximate counting for $k$-DNF. In Proc. 7th International Workshop on Approximation Algorithms for Combinatorial Optimization Problems (APPROX 2004), Lecture Notes in Computer Science, vol. 3122, Springer, pp. 417-426, 2004. 1.1, 1.2, 4

School of Computer Science, Tel Aviv University, Tel Aviv 69978, Israel

E-mail address: nachumd@tau.ac.il

School of Computer Science, Tel Aviv University, Tel Aviv 69978, Israel

E-mail address: tzameret@tau.ac.il 